\title{
Relationships Between Bifurcation and Numerical Analyses for Ignition of Hydrogen-Air Diffusion Flames
}

\author{
A. L. SÁNCHEZ, ${ }^{*}$ G. BALAKRISHNAN, ${ }^{\dagger}$ A. LIÑÁN, ${ }^{\ddagger}$ and F. A. WILLIAMS ${ }^{\S}$ \\ Center for Energy and Combustion Research, Department of Applied Mechanics and Engineering Sciences, \\ MC 0411, University of Califormia San Diego, La Jolla, CA 92093-0411 USA
}

\begin{abstract}
Linear bifurcation and numerical techniques are employed to determine critical conditions for ignition in steady, counterflow, nonpremixed hydrogen-air systems, with varying degrees of nitrogen dilution of the fuel, at temperatures larger than the crossover temperature associated with the second explosion limit for hydrogen. Analysis of profiles of the radical pool at ignition reveals that, irrespective of the degree of dilution of the fuel or oxidizer streams, the $\mathrm{O}$-atom steady state fails on the oxidizer side of the mixing layer. Therefore, at least three overall steps, with $\mathrm{O}$ and $\mathrm{H}$ atoms as the chain-branching species, are necessary to describe the ignition process. A simplified model with variable density, specific heat and transport properties, and with Stefan-Maxwell approximations for the diffusion velocities, is proposed to describe the structure of the $\mathrm{H}_{2}-\mathrm{O}_{2}-\mathrm{N}_{2}$ weakly reactive mixing layer. Results of bifurcation analysis with this flow-field model and a three-step reduced chemical-kinetic scheme show excellent agreement with results of numerical integration of the full conservation equations with detailed chemistry for all degrees of dilution of the fuel feed.
\end{abstract}

\section{INTRODUCTION}

Autoignition of hydrogen in nonpremixed environments is a complicated process for which a thorough understanding is not yet available. The process is relevant, for example, in propulsion applications that employ supersonic combustion [1]. Numerical [2-5], asymptotic [6] and bifurcation [7] analyses of ignition in counterflow systems have recently been conducted. like the present investigation, all of these studies are restricted to steady flows, for which ignition times cannot be determined but critical conditions are obtained for autoignition to occur. Although ignition is an unsteady process, the determination of criticality conditions is an essential first step that is often of direct practical usefulness in identifying necessary design parameters for combustors. Most of the work on hydrogen-air ignition has been numerical, probably because at the current level

\footnotetext{
- Graduate Student. Corresponding author. Current Address: Area de Mecánica de Fluidos, Escuela Politécnica Superior, Universidad Carlos III de Madrid, Leganes, 28911, Spain.

' Current Address: Department of Mechanical Engineering, Virginia Polytechnic Institute and State University, Blacksburg, VA 24061-0238.

' Visiting Professor. Permanent Address: ETSI Aeronaúticos, UPM, Madrid, Spain.

- Professor of Engineering Physics and Combustion.
}

of code development, full numerical integrations are relatively easy to perform for systems having so few elementary chemical steps as hydrogen-oxygen. Studies with strong analytical components are more difficult and timeconsuming because of the complexity of the mathematical analysis required. However, such investigations are important because they expose useful simplifications and show how accurate these simplifications are, thereby greatly contributing to our understanding. Following earlier work [6,7], the present investigation is of this general mathematical character.

Problems concerning chemical reactions in nonpremixed systems can be divided into two distinct categories $[8,9]$, namely, problems of the evolution type and quasisteady problems. The former are described by ordinary differential equations with boundary conditions at one point or by parabolic partial differential equations and have a unique solution that represents the evolution of the flow field as time (or a spatial time-like coordinate) progresses. Quasisteady problems, on the other hand, such as that considered here, obey ordinary differential equations with boundary conditions at two points or elliptic partial differential equations and may exhibit a multiplicity of solutions for certain ranges of the flow parameters. This multiplicity is a complicating characteristic of quasisteady problems. One aspect of the multi- 
plicity, namely, the existence of critical conditions for ignition to occur, is addressed here.

When the ignition process is dominated by a branched-chain kinetic mechanism, as it is for high-temperature hydrogen-oxygen mixtures, the distinct nature of the two types of problems results in another, more subtle difference. Since the rates of the chain-branching and chain-terminating steps are proportional to the concentrations of intermediate species, in the absence of radicals the only chemical activity is that associated with the initiation steps, whose rates (proportional to concentrations of reactants) are usually very small in high-temperature ignition of hydrogen [10] or hydrocarbons. This enables initiation steps to be neglected entirely for quasisteady problems, but for evolution problems beginning purely with reactants, the effect of initiation must be included if the solution is to evolve from frozen conditions as time progresses, unless a nonzero initial concentration of radicals in the flow field is imposed artificially. The latter crutch was employed in the study of modeled branched-chain ignition in two flows of interest, a premixed system exposed to a hot inert gas [11] and a supersonic mixing layer [12]. By way of contrast, the initiation steps were retained by Trevin̄o and Méndez [13] in the analysis of ignition of a stream of hydrogen and oxygen by a hot plate and more recently by Ju and Niioka [14] in a numerical investigation of ignition in the nonpremixed hydrogen-air supersonic mixing layer. It is worth remarking that, in some situations [15], a parabolic problem may be transformed into an elliptic one, and use of the method presented below can be made, even though the problem originally is of the evolution type.

In the analysis of quasisteady problems, inclusion of an initiation step is unnecessary for finding nontrivial solutions. If only the chainbranching and chain-terminating steps are retained in the kinetic mechanism, then all chemical production terms in the conservation equations are proportional to concentrations of radicals, and there always exists a solution corresponding to frozen flow with zero radical concentrations [7]. If the different solutions to the quasisteady problem are exhibited in a plot of a representative maximum radical concen- tration, $Y_{R \max }$, as a function of a Damköhler number, $\Delta$, defined as the ratio of a characteristic convective or diffusive time to a characteristic chemical time, then this first solution lies along the horizontal axis. At a critical value of the Damköhler number, $\Delta_{c}$, the frozen solution loses stability, and a second branch of solutions, corresponding to ignited states, bifurcates from it.

The shape of the bifurcated branch depends on the geometry of the particular flow field considered as well as on the chemical-kinetic mechanism. Typically, the resultant loci of solutions correspond to one of the two curves sketched in Fig. 1, where physically unacceptable solutions with negative $Y_{R \text { max }}$ have been included for illustrative purposes. These types of curves have been described [16] as imperfect supercritical bifurcations, with a transcritical bifurcation at $\Delta=\Delta_{c}$. More complicated behaviors arise when ignition takes place in premixed systems or in the presence of walls [17, 18]. If attention is restricted to nonnegative values of $Y_{R \text { max }}$, then the curves locally resem-

a)

b)

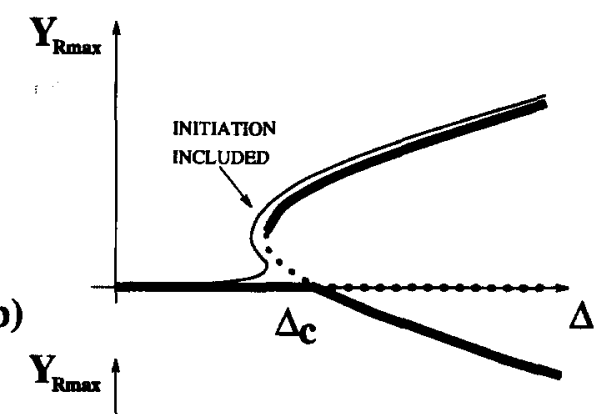

Fig. 1. Schematic representation of the dependence of maximum radical concentration on the value of the Damköhler number corresponding to different solutions for quasisteady nonhomogeneous systems with chainbranching chemistry. 
ble those for an ordinary subcritical bifurcation in Fig. 1a and for an ordinary supercritical bifurcation in Fig. 1b, a terminology that was employed previously [7] for simplicity.

The slope of the transcritical bifurcation at the bifurcation point depends on the competition between reactant consumption and chemical heat generation [7]. If the ignition reactions are sufficiently exothermic, then the bifurcated solution possesses a negative slope, as shown in Fig. 1a, and the ignited branch evolves to reproduce the S-shape curve usually encountered in diffusion flames, the autoignition transition at $\Delta=\Delta_{c}$ being abrupt. On the other hand, when the exothermicity is small enough that reactant consumption dominates the ignition process, the bifurcation slope at $\Delta=\Delta_{c}$ is positive, as shown in Fig. 1b, and the resultant ignited branch corresponds to a smooth transition from the frozen state to a diffusion-flame solution, with the value of $\Delta_{c}$ determining conditions at which chemical reaction begins to take place. Inclusion of an initiation step in the kinetic mechanism would slightly distort this picture, i.e., the frozen state would no longer be a solution to the problem; therefore, according to the strict definition, no bifurcation would exist, as illustrated by the lightweight curves in Fig. 1.

The value of $\Delta_{c}$ and the slope of the transcritical bifurcation can be found by means of a linear and weakly nonlinear perturbation analysis around $\Delta=\Delta_{c}$. This method was applied for high-temperature ignition [7] to explain the different shapes that the ignition curves may exhibit. Two distinct temperatures were found (1) characterize the ignition behavior, the crossover temperature $T_{c}$, at which the rates of production and consumption of $\mathrm{H}$ atoms are equal, and a transition temperature $T_{s}>T_{c}$, which separates ignition processes dominated by heat release from those dominated by reactant consumption. For temperatures of the oxidizer stream $T_{\infty}$ below $T_{c}$, the frozen solution was found to be stable for all values of the Damköhler number, so that no branched-chain explosion is possible, and the ignited branch appears as a C-shape curve unconnected to the frozen solution. For temperatures above crossover, the net production of $\mathrm{H}$ atoms is positive, and a chain-branching explosion de- velops when the value of the Damköhler number reaches a critical value. For $T_{x}$ in the range $T_{c}<T_{x}<T_{s}$, the effect of chemical heat release, mainly provided by the radical-recombination reaction $\mathrm{H}+\mathrm{O}_{2}+\mathrm{M} \rightarrow \mathrm{HO}_{2}+\mathrm{M}$, is dominant, and the ignition behavior corresponds to that shown in Fig. 1a. As the temperature increases, the specific reaction-rate constant for this recombination decreases, while those for chain branching increase rapidly, causing the rate of the recombination step to decrease relative to that of chain branching. For sufficiently large values of $T_{x}$ the resulting temperatures rise is so small that oxygen consumption becomes the dominant factor, and the criticality of the bifurcation changes. A smooth transition behavior, i.e., no abrupt ignition, as in Fig. 1b, is obtained for $T_{\infty}$ above $T_{s}$. A simple analytic expression, determined with two-step reduced chemistry and with a simplified constant-density model adopted for the flow field, was provided for $T_{s}$. Although the results obtained in Ref. 7 showed qualitative agreement with numerical studies, quantitative comparisons were not made.

In this paper, a linear bifurcation analysis will be performed to obtain the critical Damköhler number at which the ignition branch bifurcates from the frozen solution, which determines either conditions for abrupt ignition $\left(T_{\infty}<T_{s}\right)$ or conditions at which chemical reaction starts taking place $\left(T_{\infty}>T_{s}\right)$. Calculations will be made to test the accuracy with which bifurcation methods predict the critical conditions for ignition in problems of the quasisteady type. A weakly nonlinear analysis, analogous to the one presented previously [7], would be required to discriminate between the two types of behavior, but such analysis will not be presented here because we have found that, to achieve good agreement with results of numerical integrations, the analysis would become undesirably complicated and would present difficulties in numerical integration much greater than those with full chemistry. Except when the fuel stream is very diluted, the effects of large variations of density, transport properties and strain rate across the mixing layer and of non-Fickian diffusion must be taken into account in the bifurcation analysis if accurate results are to be obtained, and there- 
fore the simplifications that were made in our previous work [7] can no longer be retained. A simplified description that includes variable density and Stefan-Maxwell transport therefore will be developed here for the $\mathrm{H}_{2}-\mathrm{O}_{2}-\mathrm{N}_{2}$ weakly reactive mixing layer. Although Stefan-Maxwell transport descriptions can be found in previous analytical studies of premixed flames $[19,20]$, their application to the analysis of nonpremixed ignition is novel. Thermal diffusion, which can have significant influences under some conditions [5, 21, 22], is relatively inconsequential in the situations addressed here and is excluded from the analysis.

It is known $[7,15]$ that at least a three-step reduced chemical-kinetic mechanism (with $\mathrm{H}$ and $\mathrm{O}$ as chain-branching species) is needed for the chemistry description to be consistent everywhere in the flow field. Isothermal solutions obtained with this kinetic scheme will be shown to compare well with those obtained by numerical integration of the complete conservation equations with detailed transport and chemistry. Comparisons with results obtained for other flowfield and chemistry descriptions also will be presented. It will be shown that use of a simpler chemical-kinetic mechanism, with $O$ in steady state everywhere, introduces significant inaccuracies for all dilutions. Reasons for this failure will be identified by consideration of the asymptotic structure of the radical pool at ignition.

\section{KINETIC MECHANISM}

The 21-step chemical-kinetic scheme with the rate parameters shown in Table 1 was selected from the literature $[1,23-27]$ as the detailed chemical mechanism for the numerical integrations of the full conservation equations. The rationale behind this selection has been presented previously [1]. Simplifications to this mechanism are needed for the bifurcation analysis.

Ignition in homogeneous mixtures was studied by Treviño [10], who found that, at high temperatures, the concentration of $\mathrm{H}_{2} \mathrm{O}_{2}$ is negligible. He worked with a set of seven elementary reactions (the forward reactions 1-6 and backward reaction 6) among the species $\mathrm{H}_{2}, \mathrm{O}_{2} \mathrm{H}, \mathrm{O}, \mathrm{OH}, \mathrm{HO}_{2}$, and $\mathrm{H}_{2} \mathrm{O}$ to describe the ignition process. The validity of this mechanism for the description of ignition in nonpremixed environments was tested numerically in a recent study [14] and found to give ignition lengths within $5 \%$ of those obtained with detailed chemistry. Hence, it is reasonable to adopt Treviño's seven-step description as a starting point, as is done here.

For quasisteady problems, this scheme can be further simplified by neglecting the initiation step, $\mathrm{H}_{2}+\mathrm{O}_{2} \rightarrow \mathrm{HO}_{2}+\mathrm{H}$, whose effect is negligible once trace amounts of radicals are present, and by assuming that the hydroperoxyl radicals are in steady state $[5,10,13,30]$. This steady-state assumption for $\mathrm{HO}_{2}$ has been shown to be a valid approximation for the description of $\mathrm{H}_{2}-\mathrm{O}_{2}$ ignition in nonpremixed environments in a number of previous numerical studies [5, 14], the reason behind the high accuracy of this approximation being the fact that the characteristic reaction time for the production step $\mathrm{H}+\mathrm{O}_{2}+\mathrm{M} \rightarrow \mathrm{HO}_{2}+\mathrm{M}$ is much larger than the corresponding times for the consumption steps (mainly $\mathrm{HO}_{2}+\mathrm{H} \rightarrow$ $\mathrm{OH}+\mathrm{OH}$ ), giving a small hydroperoxyl concentration. From a fundamental point of view, it is worth emphasizing that, since the dependence of the production rate on the radical concentration is linear, while that of the consumption steps is quadratic, these approximations cannot be exactly valid extremely close to the bifurcation point, where the $\mathrm{HO}_{2}$ steady state necessarily breaks down. However, the reaction-rate constant corresponding to $\mathrm{H}+$ $\mathrm{O}_{2}+\mathrm{M} \rightarrow \mathrm{HO}_{2}+\mathrm{M}$ (divided by the thirdbody concentration) is so much smaller than that of $\mathrm{HO}_{2}+\mathrm{H} \rightarrow \mathrm{OH}+\mathrm{OH}$ that the steady state already is reached when trace amounts of radicals are present. Relaxation of the $\mathrm{HO}_{2}$ steady-state assumption in a recent numerical study [4] has indicated that ignition behaviors more complicated than those presented in Fig. 1 may develop when complex chemistry is considered. However, the complex behaviors reported [4] emerge for negligibly small values of the radical concentrations, with $\mathrm{H}$ mole fractions typically being $10^{-9}$. These minute concentrations are comparable with the radical content of ordinary humid air at temperatures typical of mixing-layer ignition, which causes the reported ignition complexity to become an 
TABLE 1

Rate Parameters for the Hydrogen-Oxygen System Adopted in the Present Study [1]; the Specific Reaction-Rate Constants $k_{i}$ Vary with Temperature $T$ According to $k_{i}=A_{i} T^{n_{i}} \exp \left(-E_{i} / R^{o} T\right)$.

\begin{tabular}{|c|c|c|c|c|c|}
\hline No. & Reactions & $A^{a}$ & $n^{a}$ & $E^{a}$ & Source \\
\hline 1 & $\mathrm{H}+\mathrm{O}_{2} \rightleftharpoons \mathrm{OH}+\mathrm{O}$ & $3.52 \times 10^{16}$ & -0.7 & 17070 & Mansten et al. [28] \\
\hline 2 & $\mathrm{H}_{2}+\mathrm{O} \rightleftharpoons \mathrm{OH}+\mathrm{H}$ & $5.06 \times 10^{4}$ & 2.67 & 6290 & Yetter et al. [29] \\
\hline $3^{b}$ & $\mathrm{H}+\mathrm{O}_{2}+\mathrm{M} \rightleftharpoons \mathrm{HO}_{2}+\mathrm{M}$ & $6.76 \times 10^{19}$ & -1.42 & 0 & Yetter et al. [29] \\
\hline 4 & $\mathrm{H}_{2}+\mathrm{OH} \rightleftharpoons \mathrm{H}_{2} \mathrm{O}+\mathrm{H}$ & $1.17 \times 10^{9}$ & 1.3 & 3626 & Baulch et al. [23] \\
\hline 5 & $\mathrm{H}+\mathrm{HO}_{2} \rightleftharpoons \mathrm{OH}+\mathrm{OH}$ & $1.70 \times 10^{14}$ & 0.0 & 874 & Baulch et al. [23] \\
\hline 6 & $\mathrm{H}+\mathrm{HO}_{2} \rightleftharpoons \mathrm{H}_{2}+\mathrm{O}_{2}$ & $4.28 \times 10^{13}$ & 0.0 & 1411 & Baulch et al. [23] \\
\hline 7 & $\mathrm{OH}+\mathrm{OH} \rightleftharpoons \mathrm{H}_{2} \mathrm{O}+\mathrm{O}$ & \multicolumn{3}{|c|}{$k=5.46 \times 10^{11} \exp (0.00149 T)$} & Yetter et al. [29] \\
\hline 8 & $\mathrm{OH}+\mathrm{HO}_{2} \rightleftharpoons \mathrm{H}_{2} \mathrm{O}+\mathrm{O}_{2}$ & $2.89 \times 10^{13}$ & 0.0 & -497 & Baulch et al. [23] \\
\hline $\mathbf{y}^{\prime}$ & $\mathrm{H}+\mathrm{H}+\mathrm{M} \rightleftharpoons \mathrm{H}_{2}+\mathrm{M}^{2}$ & $1.80 \times 10^{18}$ & -1.0 & 0 & Smooke [24] \\
\hline $10^{b}$ & $\mathrm{H}+\mathrm{OH}+\mathrm{M} \rightleftharpoons \mathrm{H}_{2} \mathrm{O}+\mathrm{M}$ & $2.20 \times 10^{22}$ & -2.0 & 0 & Baulch et al. [23] \\
\hline 11 & $\mathrm{HO}_{2}+\mathrm{HO}_{2} \rightleftharpoons \mathrm{H}_{2} \mathrm{O}_{2}+\mathrm{O}_{2}$ & $3.02 \times 10^{12}$ & 0.0 & 1390 & Yetter et al. [29] \\
\hline $12^{d}$ & $\mathrm{H}_{2} \mathrm{O}_{2}+\mathrm{M} \rightleftharpoons \mathrm{OH}+\mathrm{OH}+\mathrm{M}$ & $1.20 \times 10^{17}$ & 0.0 & 45500 & Yetter et al. [29] \\
\hline 1.3 & $\mathrm{H}_{2} \mathrm{O}_{2}+\mathrm{OH} \rightleftharpoons \mathrm{H}_{2} \mathrm{O}+\mathrm{HO}_{2}$ & $7.08 \times 10^{12}$ & 0.0 & 1430 & Yetter et al. [29] \\
\hline 14 & $\mathrm{O}+\mathrm{HO}_{2} \rightleftharpoons \mathrm{OH}+\mathrm{O}_{2}$ & $2.00 \times 10^{13}$ & 0.0 & 0 & Baulch et al. [23] \\
\hline 15 & $\mathrm{H}+\mathrm{HO}_{2} \rightleftharpoons \mathrm{O}+\mathrm{H}_{2} \mathrm{O}$ & $3.10 \times 10^{13}$ & 0.0 & 1720 & Baulch et al. [23] \\
\hline $16^{b}$ & $\mathrm{H}+\mathrm{O}+\mathrm{M} \rightleftharpoons \mathrm{OH}+\mathrm{M}$ & $6.20 \times 10^{16}$ & -0.6 & 0 & Yetter et al. [29] \\
\hline $17^{b}$ & $\mathrm{O}+\mathrm{O}+\mathrm{M} \rightleftharpoons \mathrm{O}_{2}+\mathrm{M}$ & $6.17 \times 10^{15}$ & -0.5 & 0 & Yetter et al. [29] \\
\hline 18 & $\mathrm{H}_{2} \mathrm{O}_{2}+\mathrm{H} \rightleftharpoons \mathrm{H}_{2} \mathrm{O}+\mathrm{OH}$ & $1.00 \times 10^{13}$ & 0.0 & 3590 & Smooke [24] \\
\hline 19 & $\mathrm{H}_{2} \mathrm{O}_{2}+\mathrm{H} \rightleftharpoons \mathrm{HO}_{2}+\mathrm{H}_{2}$ & $4.79 \times 10^{13}$ & 0.0 & 7950 & Smooke [24] \\
\hline 20 & $\mathrm{O}+\mathrm{OH}+\mathrm{M} \rightleftharpoons \mathrm{HO}_{2}+\mathrm{M}$ & $1.00 \times 10^{16}$ & 0.0 & 0 & Smooke [24] \\
\hline 21 & $\mathrm{H}_{2}+\mathrm{O}_{2} \rightleftharpoons \mathrm{OH}+\mathrm{OH}$ & $1.70 \times 10^{13}$ & 0.0 & 47780 & Smooke [24] \\
\hline
\end{tabular}

${ }^{a}$ Units: $\mathrm{mol} / \mathrm{cm}^{3}, \mathrm{~s}^{-1}, \mathrm{~K}, \mathrm{cal} / \mathrm{mol}$; rates for reverse steps obtained from JANAF thermochemical equilibrium data.

${ }^{b}$ Chaperon efficiencies: $\mathrm{H}_{2}: 2.5, \mathrm{H}_{2} \mathrm{O}: 12.0, \mathrm{O}_{2}: 1.0$ and $\mathrm{N}_{2}: 1.0$.

${ }^{c}$ Chaperon efficiencies: $\mathrm{H}_{2}: 1, \mathrm{H}_{2} \mathrm{O}: 6.5, \mathrm{O}_{2}: 0.4$ and $\mathrm{N}_{2}: 0.4$.

${ }^{d}$ Chaperon efficiencies: $\mathrm{H}_{2}: 2.5, \mathrm{H}_{2} \mathrm{O}: 15.0, \mathrm{O}_{2}: 1.0$ and $\mathrm{N}_{2}: 1.0$.

irrelevant detail of the complete branch of ignited solutions. Hence, although it is true that the steady-state assumption for $\mathrm{HO}_{2}$ does not strictly hold extremely near the ignition point, this steady state is readily recovered for negligibly small values of the radical concentration, so that for practical purposes the description of the ignition branch does not require consideration of effects of $\mathrm{HO}_{2}$ departure from the steady state.

For mixtures that are not very lean, $\mathrm{O}$ and $\mathrm{OH}$ can also be assumed to be in steady state [10], which simplifies the mechanism to a twostep reduced scheme. This two-step description has been employed in the previous bifurcation analysis of nonhomogeneous ignition [7] and elsewhere $[30,31]$. While this mechanism consistently holds for premixed systems that are not too lean, its application to nonhomogeneous ignition presents a fundamental difficulty. As previously pointed out [7], far on the oxidizer side the mixture necessarily becomes very lean, which causes the mechanism to fail.
It was shown that careless application of this kinetic scheme everywhere in the mixing layer results in negative fuel concentrations in some regions on the oxidizer side. This inconsistency prevents this simple mechanism from being used in this form in numerical calculations, since no realistic solutions of the conservation equations exist. The difficulty was previously overcome [7] by employing the two-step mechanism only up to the point at which the $\mathrm{H}_{2}$ concentration vanishes and by freezing the chemical reactions beyond that point. This consistently describes the structure of the mixing layer and reproduces the flame-sheet approximation in the limit of infinite Damköhler number.

The validity of the two-step reduced mechanism mentioned above extends from the fuel stream across the mixing layer to a location where the mixture becomes so lean that the rates of the reactions $\mathrm{H}_{2}+\mathrm{O} \rightarrow \mathrm{OH}+\mathrm{H}$ and $\mathrm{H}_{2}+\mathrm{OH} \rightarrow \mathrm{H}_{2} \mathrm{O}+\mathrm{H}$, proportional to the fuel concentration, are too small to sustain the 
steady states of the $\mathrm{O}$ and $\mathrm{OH}$ radicals. Since the reaction-rate constant of the $\mathrm{O}$-consuming reaction is roughly four times smaller than that of the OH-consuming step, the steady-state assumption for $\mathrm{O}$ fails first, while that for $\mathrm{OH}$ holds farther into the oxidizer stream. Therefore relaxing the steady-state assumption for $\mathrm{O}$, rather than that for $\mathrm{OH}$, is the appropriate first step towards improving the kinetic description. This explains why results of numerical integrations obtained by using $\mathrm{H}$ and $\mathrm{OH}$ as the chain-branching species are rather poor compared with those obtained when $\mathrm{H}$ and $\mathrm{O}$ are the two species not put into steady state [5, 14].

Neglecting the initiation step and assuming $\mathrm{HO}_{2}$ and $\mathrm{OH}$ to be in steady state reduces Treviño's seven-step scheme to the three global steps [32]

$$
\begin{aligned}
& \mathrm{H}_{2}+\mathrm{O}_{2} \rightarrow \mathrm{O}+\mathrm{H}_{2} \mathrm{O}, \\
& \mathrm{O}+2 \mathrm{H}_{2} \rightarrow 2 \mathrm{H}+\mathrm{H}_{2} \mathrm{O}, \\
& 2 \mathrm{H}+\mathrm{M} \rightarrow \mathrm{H}_{2}+\mathrm{M},
\end{aligned}
$$

with rates given by $\omega_{1}=\omega_{1}+(1-\alpha) \omega_{3}, \omega_{11}$ $=\omega_{2}+(1-\alpha) \omega_{3}$ and $\omega_{\text {III }}=\omega_{3}$. Here, $\omega_{1}$, $\omega_{2}$, and $\omega_{3}$ are, respectively, the rates of the forward elementary reactions

$$
\begin{aligned}
& \mathrm{H}+\mathrm{O}_{2} \stackrel{1}{\rightarrow} \mathrm{OH}+\mathrm{O}, \\
& \mathrm{H}_{2}+\mathrm{O} \stackrel{2}{\rightarrow} \mathrm{OH}+\mathrm{H}, \\
& \mathrm{H}+\mathrm{O}_{2}+\mathrm{M} \stackrel{3}{\rightarrow} \mathrm{HO}_{2}+\mathrm{M},
\end{aligned}
$$

and $\left(\alpha^{-1}-1\right)$ denotes the ratio of the rate of the elementary step $\mathrm{HO}_{2}+\mathrm{H} \rightarrow \mathrm{OH}+\mathrm{OH}$ (o) that of the step $\mathrm{HO}_{2}+\mathrm{H} \rightarrow \mathrm{H}_{2}+\mathrm{O}_{2}$. It can be shown from the data in Table 1 that $\alpha=1 / 6$ over the temperature range of interest $\left(900<T_{\infty}<1500\right)$.

A more accurate description arises by relaxing the steady-state assumption for $\mathrm{OH}$ as well $[5,14]$. However, the relatively small gain in accuracy that would be obtained does not justify the additional analytical complexity that would result from adding another overall step to the scheme, and therefore such possible improvement is not considered in the present paper.
FORMULATION AND PROCEDURE FOR FULL NUMERICAL INTEGRATION

A steady, axisymmetric, ideal-gas, counterflow configuration is considered, with hydrogen diluted with nitrogen flowing from $y=-\infty$ and air from $y=\infty$. Here, $x$ and $y$ denote the radial and axial coordinates, while $u$ and $v$ are the radial and axial velocity components. Conditions in the inviscid, irrotational outer flows of fuel and oxidizer are denoted by the subscripts $-\infty$ and $\infty$, respectively, and the outer radial velocity is assumed to be proportional to the distance from the axis, i.e., $u_{ \pm \infty}=a_{ \pm \infty} x$, where $a_{ \pm \infty}$ is the strain rate in the outer stream at $\pm \infty$. For this problem, the conservation equations with $N$ different chemical species reduce to the following set of ordinary differential equations [33-35]:

Continuity:

$\frac{d V}{d y}+2 \rho U=0$.

$x$-Momentum:

$V \frac{d U}{d y}=-\rho U^{2}+\rho_{\infty} a_{\infty}^{2}+\frac{d}{d y}\left(\mu \frac{d U}{d y}\right)$.

$y$-Momentum:

$$
\frac{d p}{d y}=0 \text {. }
$$

Species:

$V \frac{d Y_{i}}{d y}=-\frac{d}{d y}\left(\rho Y_{i} V_{i}\right)+w_{i}$.

Energy:

$$
\begin{aligned}
V c_{p} \frac{d T}{d y}= & \frac{d}{d y}\left(\lambda \frac{d T}{d y}\right)-\sum_{i=1}^{N} h_{i} w_{i} \\
& -\rho \sum_{i=1}^{N} c_{p i} Y_{i} V_{i} \frac{d T}{d y} .
\end{aligned}
$$

In these equations, we have introduced for convenience the functions $U$ and $V$, defined as $u=U x$ and $V=\rho v$. The temperature, pressure, density, thermal conductivity, viscosity, and specific heat at constant pressure of the 
mixture are denoted by $T, p, \rho, \lambda, \mu$, and $c_{p}=\sum_{t}^{N} Y_{i} c_{p i}$, respectively, while $Y_{i}, V_{i}, w_{i}$ and $c_{n}$ are the mass fraction, diffusion velocity, mass rate of production and specific heat at constant pressure of species $i$. Here, $h_{i}=h_{i}^{T}$ $+h_{i}^{o}$ is the enthalpy of species $i$, with $h_{i}{ }^{T}=$ $i_{1}{ }^{T}{ }^{T} c_{p i} d T$ and $h_{i}{ }^{o}$ identifying, respectively, its thermal enthalpy and its enthalpy of formation, and $T^{\circ}$ being a fixed, standard reference temperature. Equations 1-5 are subject to the boundary conditions

$$
\begin{array}{r}
U=a_{\infty}, \quad V=\rho_{\infty} v_{\infty}, \quad Y_{i}=Y_{i \infty}, \quad T=T_{\infty} \\
\text { as } y \rightarrow \infty
\end{array}
$$

and

$$
\begin{aligned}
& U=a_{-\infty}=\left(\rho_{-\infty} / \rho_{\infty}\right)^{1 / 2} a_{\infty}, \quad V=\rho_{-\infty} v_{-\infty}, \\
& Y_{1}=Y_{i-\infty}, \quad T=T_{-\infty} \quad \text { as } y \rightarrow-\infty,
\end{aligned}
$$

and must be supplemented with the ideal gas law

$\rho=\frac{p \bar{W}}{R^{o} T}$,

where $R^{o}$ is the universal gas constant and $\bar{W}=\left(\sum_{i=1}^{N} Y_{i} / W_{i}\right)^{-1}$ is the mean molecular weight, while $W_{i}$ denotes the molecular weight of species $i$.

These equations were integrated numerically by discretizing the spatial differential operators on a mesh of finite length using a central difference stencil for the derivatives. To obtain good starting estimates for stiff problems, the numerical code has the option of evaluating the convective terms using a first-order upwind method. In order to ensure proper accuracy, adaptive spatial grids were used, and the resulting system of nonlinear algebraic equations was then solved by a Newton-like method. Modifications to the basic algorithm are introduced in the vicinity of singular points, i.e., turning points of the S-shape curve, as described elsewhere [36]. A set of multicomponent transport equations for $V_{i}$ is employed in the numerical integrations, with transport properties obtained from the vectorized version of CHEMKIN [35], which uses NASA polynomial fits to evaluate the thermodynamic and transport data for the multicomponent mixture, with thermal diffusion excluded here.
As stated earlier, the numerical integrations of the conservation equations employed the 21-step kinetic mechanism shown in Table 1 to describe the chemistry. Because of the presence of initiation steps, such as $\mathrm{H}_{2}+\mathrm{O}_{2} \rightarrow$ $\mathrm{HO}_{2}+\mathrm{H}$, smooth curves, rather than abrupt bifurcations, are obtained when the solutions found by numerical integration of the equations with detailed chemistry are presented in a diagram of $Y_{R \max }$ as a function of $a_{\infty}{ }^{-1}$. In order to compare these results with those obtained from the linear bifurcation analysis, the critical point at which the ignited branch bifurcates must be identified. While the turning point itself is a suitable definition of the critical strain rate when $T_{\infty}<T_{s}$, no such clearcut rule exists to identify critical conditions when a smooth transition of the type shown in Fig. 1b takes place. The specific criterion chosen here in the absence of abrupt ignition is that the critical strain rate corresponds to the solution for which the peak mass fraction of $\mathrm{H}$ atoms equals $10^{-6}$. Ignition results are not very sensitive to these selections, in that critical values change by less than $5 \%$ if $10^{-6}$ is replaced by $10^{-5}$ or $10^{-7}$ or if the turning point is replaced by an extrapolation to the frozen curve.

The conservation equations can be simplified by introducing a nondimensional density-weighted coordinate [37], $\eta=\left(2 a_{\infty} \rho_{\infty} /\right.$. $\left.\mu_{\infty}\right)^{1 / 2} \int_{0}^{y} \bar{\rho} d y$, and a nondimensional stream function $F(\eta)$, such that $U=a_{\infty} F^{\prime}(\eta)$. In the notation employed here the prime denotes differentiation with respect to $\eta$. The $x$ momentum, species and energy equations reduce to [32]

$$
\begin{aligned}
& \left(C F^{\prime \prime}\right)^{\prime}+F F^{\prime \prime}+\frac{1}{2}\left(\frac{1}{\bar{\rho}}-F^{2}\right)=0, \\
& -\left(\bar{\rho} Y_{i} \bar{V}_{i}\right)^{\prime}+F Y_{i}^{\prime}=-\frac{\bar{w}_{i}}{\bar{\rho}}
\end{aligned}
$$

and

$$
\begin{aligned}
& {\left[\frac{C}{\operatorname{Pr}}\left(\bar{h}^{T}\right)^{\prime}\right]^{\prime}+F\left(\bar{h}^{T}\right)^{\prime}} \\
& \quad-\left(\frac{C}{\operatorname{Pr}} \sum_{i=1}^{N} \bar{h}_{i}^{T} Y_{i}^{\prime}+\bar{\rho} \sum_{i=1}^{N} \bar{h}_{i}^{T} Y_{i} \bar{V}_{i}\right)^{\prime} \\
& \quad=\sum_{i=1}^{N} \frac{\bar{h}_{i}^{o} \bar{w}_{i}}{\bar{\rho}},
\end{aligned}
$$


where the nondimensional variables $\bar{\rho}=\rho / \rho_{x}$, $\bar{V}_{i}=\left[\rho_{x} /\left(2 a_{x} \mu_{x}\right)\right]^{1 / 2} V_{i}, \quad \bar{w}_{i}=w_{i} /\left(2 a_{x} \rho_{x}\right), \bar{h}^{T}$ $=h^{T} / h_{x}{ }^{T}$ and $\bar{h}_{i}{ }^{T}=h_{i}{ }^{T} / h_{x}{ }^{T}$, and the quantities $\bar{h}_{i}^{o}=h_{i}^{o} / h_{x}{ }^{T}$ have been introduced for convenience, with $h^{T}=\sum_{i=1}{ }^{N} h_{i}^{T} Y_{i}$ denoting the thermal enthalpy of the mixture. In these equations, $\operatorname{Pr}=\mu c_{p} / \lambda$ is the Prandtl number and $C=(\rho \mu) /\left(\rho_{x} \mu_{x}\right)$ is the Chapman-Rubesin parameter. The boundary conditions for Eqs. 8-10 can be written as $F^{\prime}(+\infty)=1, F(0)$ $=0, F^{\prime}(-\infty)=a_{-x} / a_{x}=\left(\rho_{x} / \rho_{-x}\right)^{1 / 2}, Y_{i}(\infty)$ $=Y_{i x}, Y_{i}(-\infty)=Y_{i-\infty}, h^{T}(\infty)=1$ and $\bar{h}^{T}(-\infty)$ $=h_{-\infty}{ }^{T} / h_{x}{ }^{T}$. Although the numerical integrations described above did not use this formulation, the results should be the same, since Eqs. 8-10 are simply a dimensionless version of Eqs. 1-5.

As mentioned before, a transport formulation based on variable diffusion coefficients is utilized in the numerical integrations. However, it is advantageous for analytical purposes to adopt a transport description based on binary diffusion coefficients, which are independent of the composition. If thermal and pressure-gradient diffusions are neglected, the multicomponent diffusion equations [38] that determine $\bar{V}_{i}$ reduce to the Stefan-Maxwell equations

$$
C X_{i}^{\prime}=\bar{\rho} \sum_{i=1}^{N} S_{i j} X_{i} X_{j}\left(\bar{V}_{j}-\bar{V}_{i}\right),
$$

which give implicitly the diffusion velocities for given values of the mole fractions $X_{i}=Y_{i} \bar{W} / W_{i}$ and their gradients. In these equations, $S_{i j}=$ $\mu /\left(\rho D_{i j}\right)$ is the nondimensional Schmidt number for species pair $i$ and $j$, where $D_{i j}$, independent of the mixture composition, is the binary diffusion coefficient corresponding to this species pair.

\section{SIMPLIFIED TRANSPORT DESCRIPTION}

The critical Damköhler number for ignition can be determined by solving a version of the conservation equations that is linearized about the frozen solution. Linearization around the bifurcation point is achieved by introducing expansions of the flow variables in powers of a small parameter $\epsilon$, where $\epsilon$ measures the max- imum concentration of a representative chainbranching radical. Since the chemical production terms are proportional to $\epsilon$, at leading order the problem reduces to that of frozen flow, which must be solved first to determine $F, \bar{h}^{T}, Y_{\mathrm{O}_{2}}$, and $Y_{\mathrm{H}_{2}}$ of the base solution. Expansion to first order in $\epsilon$ about this base solution provides a set of linear differential equations with homogeneous boundary conditions at $\pm \infty$ that constitutes an eigenvalue problem for determining the critical Damköhler number.

Since considerable numerical effort is required to solve this complete bifurcation problem, it is desirable to seek simplifications. Many of the most ordinary simplifying assumptions, such as constant Schmidt numbers and unity Chapman-Rubesin parameter $C$, are poor approximations because of the presence of significant amounts of the light species $\mathrm{H}_{2}$. The key observation to simplify the problem is that the composition at ignition is that of a tertiary mixture with two very similar species, $\mathrm{N}_{2}$ and $\mathrm{O}_{2}$, and a dissimilar one, $\mathrm{H}_{2}$, while the concentrations of water vapor and radicals are negligibly small. It is reasonable to assume that the molecular weights, specific heats and transport properties of $\mathrm{N}_{2}$ and $\mathrm{O}_{2}$ are equal. Under that assumption, $\rho, \mu$, and $\lambda$ correspond to those of a binary mixture and can be expressed as functions of the temperature and $Y_{\mathrm{H}_{2}}$, and the thermal enthalpy reduces to

$\bar{h}^{T}=Y_{\mathrm{H}_{2}} \bar{h}_{\mathrm{H}_{2}}^{T}+\left(1-Y_{\mathrm{H}_{2}}\right) \bar{h}_{\mathrm{N}_{2}}^{T}$.

Also, the transport description is considerably simplified. In particular, the diffusion of $\mathrm{H}_{2}$ into the mixture corresponds to that of a binary mixture and is governed by Fick's law [37]. If we let $S_{i}$ denote the Schmidt number for species pair $i$ and $\mathrm{N}_{2}$, the diffusion velocity of $\mathrm{H}_{2}$ can be written as [32]

$$
\bar{\rho} Y_{\mathrm{H}_{2}} \bar{V}_{\mathrm{H}_{2}}=-C Y_{\mathrm{H}_{2}}^{\prime} / S_{\mathrm{H}_{2}} .
$$

The diffusion of molecular oxygen and the diffusion of radicals correspond to that of a third species into a binary mixture, and their diffusion velocities can be shown from Eq. 11 
to be [32]

$\bar{\rho} Y_{i} \bar{V}_{i}=-C\left(\psi Y_{i}^{\prime}-b_{i} Y_{\mathrm{H}_{2}}^{\prime} Y_{i}\right) /\left(\phi_{i} S_{i}\right)$.

Here, $\psi$ and $\phi_{i}$ are functions of $Y_{\mathrm{H}_{2}}$, defined as

$\psi=1+(\omega-1) Y_{\mathrm{H}_{2}}$

and

$\phi_{i}=1+a_{i} Y_{\mathrm{H}_{2}}$,

and $\omega, a_{i}$ and $b_{i}$ are constants given, respectively, by

$\omega=\frac{W_{\mathrm{N}_{2}}}{W_{\mathrm{H}_{2}}}$,

$a_{i}=\frac{D_{i \mathrm{~N}_{2}}}{D_{i \mathrm{H}_{2}}} \omega-1$

and

$b_{i}=\omega\left(1-\frac{D_{\mathrm{H}_{2} \mathrm{~N}_{2}}}{D_{i \mathrm{H}_{2}}}\right)+\left(1-\frac{D_{\mathrm{H}_{2} \mathrm{~N}_{2}}}{D_{i \mathrm{~N}_{2}}}\right)$.

The values of the nondimensional transport constants, evaluated using NASA polynomial fits, are $a_{\mathrm{O}_{2}}=2.83, a_{\mathrm{H}}=7.96, a_{\mathrm{O}}=3.42, b_{\mathrm{O}_{2}}$ $=2.52, b_{\mathrm{H}}=8.51$, and $b_{\mathrm{O}}=4.95$ over the range of temperature of interest.

With this transport description, which can be utilized in general in the study of weakly reactive $\mathrm{H}_{2}-\mathrm{O}_{2}-\mathrm{N}_{2}$ mixtures, the equations that determine the frozen profiles of $F, Y_{\mathrm{H}_{2}}$ and $\bar{h}^{T}$ reduce to Eq. 8 together with

$\left(\frac{C}{S_{\mathrm{H}_{2}}} Y_{\mathrm{H}_{2}}^{\prime}\right)^{\prime}+F Y_{\mathrm{H}_{2}}^{\prime}=0$

and

$$
\begin{aligned}
& {\left[\frac{C}{\operatorname{Pr}}\left(\bar{h}^{T}\right)^{\prime}\right]^{\prime}+F\left(\bar{h}^{T}\right)^{\prime}} \\
& \quad-\left[C\left(\frac{1}{\operatorname{Pr}}-\frac{1}{S_{\mathrm{H}_{2}}}\right)\left(\bar{h}_{\mathrm{H}_{2}}^{T}-\bar{h}_{\mathrm{N}_{2}}^{T}\right) Y_{\mathrm{H}_{2}}^{\prime}\right]^{\prime} \\
& \quad=0,
\end{aligned}
$$

with $\bar{h}^{T}$ given by Eq. 12 . Similarly, Eq. 14 can be substituted into Eq. 9 to generate explicit equations for the frozen concentrations of $\mathrm{O}_{2}$ and the concentration of radicals. The problem may be solved sequentially. First, Eqs. 8, 20, and 21, independent of the $\mathrm{O}_{2}$ concentration, must be integrated simultaneously with the boundary conditions previously stated to solve for the stream function, hydrogen concentration and temperature. The equation for $Y_{\mathrm{O}_{2}}$ can then be solved separately to complete the solution for the frozen mixing layer. Finally, the equations for the first perturbations to the radical concentrations can be integrated to obtain $\Delta_{c}$.

The bifurcation problem was solved numerically for the isothermal mixing layer, for which $S_{i}=S_{i \infty} \bar{\mu} / \bar{\rho}$, and the functions $\bar{\rho}$ and $\bar{\mu} \equiv$ $\mu / \mu_{\infty}$ can be written as functions of $Y_{\mathrm{H}_{2}}$ in the form

$\bar{\rho}=\left[1+(\omega-1) Y_{\mathrm{H}_{2}}\right]^{-1}$

and

$$
\begin{aligned}
\bar{\mu}= & \frac{\sigma \omega Y_{\mathrm{H}_{2}}}{c_{\mathrm{H}_{2}}+\left(\omega-c_{\mathrm{H}_{2}}\right) Y_{\mathrm{H}_{2}}} \\
& +\frac{1-Y_{\mathrm{H}_{2}}}{1+\left(\omega c_{\mathrm{AIR}}-1\right) Y_{\mathrm{H}_{2}}}
\end{aligned}
$$

where $\sigma=\mu_{\mathrm{H}_{2}} / \mu_{\mathrm{AIR}}$ and $c_{i}=\left(1.385 \mu_{i} R^{o} T\right) /$ $\left(D_{\mathrm{H}_{2} \mathrm{~N}_{2}} P W_{i}\right)$ [38]. The following values, obtained from NASA polynomial fits, were employed in the calculations: $\sigma=0.45, c_{\mathrm{H}_{2}}=$ $1.72, c_{\mathrm{AIR}}=0.264, S_{\mathrm{O}_{2^{\infty}}}=0.74, S_{\mathrm{H}_{2} \infty}=0.19$, $S_{\mathrm{H} \infty}=0.12$, and $S_{\mathrm{O} \infty}=0.48$. Combining Eqs. 9,22 and 23 yields

$y_{\mathrm{O}_{2}}^{\prime \prime}+F_{\mathrm{O}_{2}} y_{\mathrm{O}_{2}}^{\prime}+G_{\mathrm{O}_{2}} y_{\mathrm{O}_{2}}=0$,

while these same equations for the radical concentrations, with the reduced chemicalkinetic mechanism I-III adopted for the chemistry, become

$$
\begin{aligned}
y_{\mathrm{H}}^{\prime \prime}+ & F_{\mathrm{H}} y_{H}^{\prime}+G_{\mathrm{H}} y_{\mathrm{H}} \\
& =-S_{\mathrm{H} \infty} \phi_{\mathrm{H}} \Delta_{c}\left[y_{\mathrm{H}_{2}} y_{\mathrm{O}}-\gamma y_{\mathrm{O}_{2}} y_{\mathrm{H}}\right]
\end{aligned}
$$

and

$$
\begin{aligned}
y_{\mathrm{O}}^{\prime \prime}+ & F_{\mathrm{O}} y_{\mathrm{O}}^{\prime}+G_{\mathrm{O}} y_{\mathrm{O}} \\
& =-S_{\mathrm{O} \infty} \phi_{\mathrm{O}} s \Delta_{c}\left[y_{\mathrm{O}_{2}} y_{\mathrm{H}}-y_{\mathrm{H}_{2}} y_{\mathrm{O}}\right],
\end{aligned}
$$


where

$$
\begin{aligned}
F_{1} & =F_{i}\left(F, Y_{\mathrm{H}_{2}}, Y_{\mathrm{H}_{2}}^{\prime}\right) \\
& =\frac{S_{i \infty} \phi_{i}}{\bar{\rho}} F-\left(b_{i}+\omega-1+\frac{a_{i}}{\bar{\rho} \phi_{i}}\right) \bar{\rho} Y_{\mathrm{H}_{2}}^{\prime}
\end{aligned}
$$

and

$$
\begin{aligned}
G_{i} & =G_{i}\left(F, Y_{\mathrm{H}_{2}}, Y_{\mathrm{H}_{2}}^{\prime}\right) \\
& =b_{i}\left(\frac{a_{i} \bar{\rho}}{\phi_{i}} Y_{\mathrm{H}_{2}}^{\prime}+\frac{S_{\mathrm{H}_{2}^{\infty}}}{\bar{\rho}} F\right) Y_{\mathrm{H}_{2}}^{\prime} .
\end{aligned}
$$

In this formulation, $y_{\mathrm{H}_{2}}=Y_{\mathrm{H}_{2}} / Y_{\mathrm{H}_{2}-\infty}, y_{\mathrm{O}_{2}}=$ $Y_{\mathrm{O}_{2}} / Y_{\mathrm{O}_{2} \infty}, \quad y_{\mathrm{H}}=Y_{\mathrm{H}} / Y_{\mathrm{H}_{2}-\infty}, \quad y_{\mathrm{O}}=2 Y_{\mathrm{O}} / Y_{\mathrm{O}_{2} \infty}$, $J_{c}=\rho_{\infty} Y_{\mathrm{O}_{2 \infty}} k_{1 \infty} /\left(W_{\mathrm{O}_{2}} a_{\infty}\right), r=k_{2 \infty} /\left(2 k_{1 \infty}\right), \gamma=$ $\alpha k_{3 \infty} C_{\mathrm{M}} / k_{1 \infty}$ and $s=\left(W_{\mathrm{O}_{2}} Y_{\mathrm{H}_{2}-\infty}\right) /\left(W_{\mathrm{H}_{2}} Y_{\mathrm{O}_{2} \infty}\right)$, where $C_{\mathrm{M}}$ is the efficiency-weighted sum of third-body concentrations. A typical value of $r$ at the temperatures of interest is $r=1.5$, while $s$, an appropriate oxygen-to-fuel mass ratio corresponding to the approaching streams, can take on very large values for undiluted cases $(s \simeq 70)$. The critical Damköhler number $\Delta_{c}$ is constructed as the ratio of the characteristic strain time to the characteristic chemical time associated with reaction 1 . The parameter $\gamma$ corresponds to the ratio of consumption to production rates of $\mathrm{H}$ radicals, in the limit of O) radicals in steady state [7], evaluated at $\tau=T_{\infty}$.

Equations 8 and 20 are coupled through the density and viscosity variations even for the isothermal problem and therefore must be integrated simultaneously, while Eq. 24 can be solved separately once the functions $F, Y_{\mathrm{H}_{2}}$, and $Y_{\mathrm{H}_{2}}^{\prime}$ are known. However, it is equally convenient to integrate all three equations simultaneously by means of a shooting method. Details of the method employed for this purpose can be found in [39] where a somewhat less involved version of the mixing-layer equations was integrated.

Once the stream function and reactant concentrations are obtained, the equations for the radicals can be solved. The homogeneous differential equations given in Eqs. 25 and 26 with homogeneous boundary conditions $y_{11}( \pm \infty)=0$ and $y_{0}( \pm \infty)=0$ constitute an eigenvalue problem for the critical Damköhler number, i.e., solutions exist only for a discrete set of values of $\Delta_{c}$. The Damköhler number that represents ignition conditions is the one whose associated eigenfunctions both are nonnegative functions in the domain of integration $(-\infty<\eta<\infty)$. This value corresponds to the smallest positive eigenvalue for which a solution to Eqs. 25 and 26 with the given homogeneous boundary conditions exists. It is noteworthy that, because of the inclusion of $O$ in the kinetic mechanism, all of the eigenfunctions exhibit an exponential decay as $\eta \rightarrow \infty$, while if two-step chemistry with $O$ in steady state is employed solutions with both algebraic and exponential decay are possible, and one must consider fuel depletion to discard the former, as done in [7]. Solutions to the eigenvalue problem were obtained numerically by an equilibrium finite-difference method [40] with a third-order centered-difference approximation adopted for the differential operators.

\section{COMPARISONS OF BIFURCATION AND NUMERICAL RESULTS}

\section{The Frozen Mixing Layer}

With the approximations introduced, the frozen solution for the isothermal mixing layer becomes independent of $T_{\infty}$, since the nondimensional quantities $a_{i}, b_{i}$ and $S_{i \infty}$ are constant over the temperature range of interest. Therefore, the profiles of reactant concentration and stream function depend only on $Y_{\mathrm{H}_{2}-\infty}$. Comparisons of the resultant reactant profiles, $y_{\mathrm{H}_{2}}$ $=Y_{\mathrm{H}_{2}} / Y_{\mathrm{H}_{2}-\infty}$ and $y_{\mathrm{O}_{2}}=Y_{\mathrm{O}_{2}} / Y_{\mathrm{O}_{2} \infty}$, with those obtained by numerical integration of the exact equations are presented in Fig. 2, which shows that, over a wide range of dilutions, the approximate description reproduces the structure of the mixing layer quite satisfactorily. By contrast, widely used standard approximations that employ Fick's law yield poor results. This is illustrated in Fig. 3, which compares the present results with those of two such approximations. In the first one, herein referred as the constant-density model, density and transport properties are assumed to be constant everywhere, a simplification that applies to isothermal, highly diluted mixing layers; in this case 


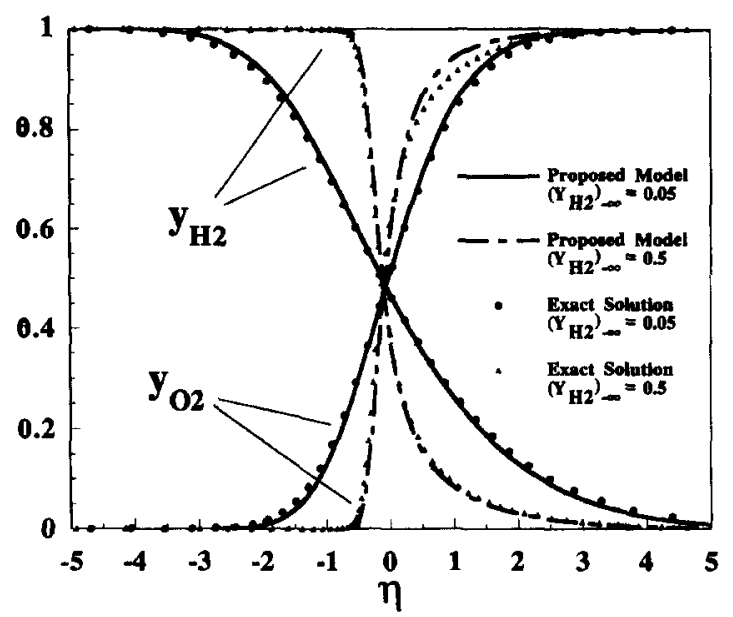

Fig. 2. The structure of the frozen mixing layer for $Y_{\mathrm{H}_{2}-\infty}$ $=0.05$ and $Y_{\mathrm{H}_{2-\infty}}=0.5$ obtained from the proposed simplified model (lines) and from the complete conservation equations at $T_{\infty}=1400 \mathrm{~K}$ (symbols).

integration of the species conservation equations with $\bar{w}_{i}=0$ yields for $Y_{\mathrm{H}_{2}}$ and $Y_{\mathrm{O}_{2}}$ the expressions

$\frac{Y_{\mathrm{H}_{2}}}{Y_{\mathrm{H}_{2}-\infty}}=\frac{1}{2} \operatorname{erfc}\left[\left(\frac{S_{\mathrm{H}_{2} \infty}}{2}\right)^{1 / 2} \eta\right]$

and

$$
\frac{Y_{\mathrm{O}_{2}}}{Y_{\mathrm{O}_{2}}}=1-\frac{1}{2} \operatorname{erfc}\left[\left(\frac{S_{\mathrm{O}_{2} \infty}}{2}\right)^{1 / 2} \eta\right] \text {. }
$$

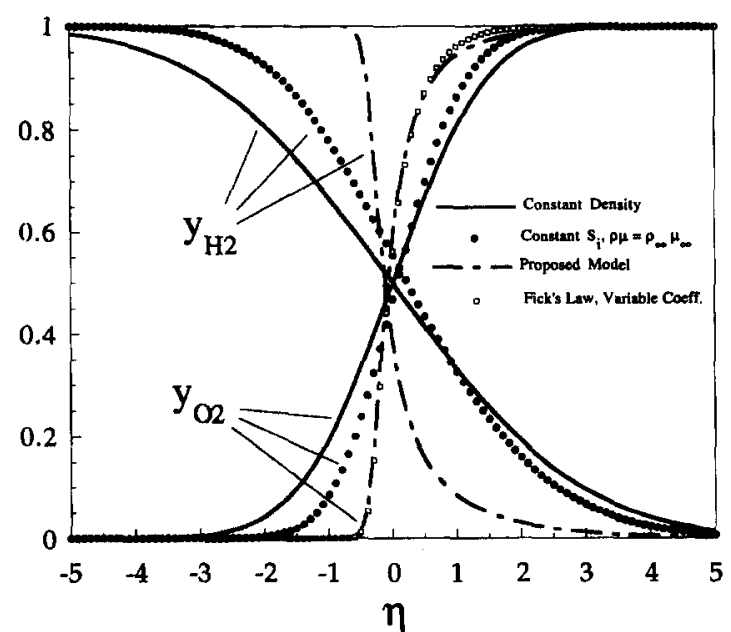

Fig. 3. The variation of $\mathrm{H}_{2}$ and $\mathrm{O}_{2}$ mass fractions with $\eta$ for $Y_{\mathrm{H}_{2}-\infty}=0.5$ as obtained from two standard approximations often used for the flow field and from the proposed simplified model with both Stefan-Maxwell and Fick descriptions adopted for the transport of oxygen.
The other assumes a Chapman-Rubesin parameter of unity, i.e., $C=1$, along with constant Schmidt numbers, but includes density variations according to $\mathrm{Eq}$. 22; the stream function and reactant concentration are determined by integrating numerically the equations

$F^{\prime \prime \prime}+F F^{\prime \prime}+\frac{1}{2}\left(\frac{1}{\bar{\rho}}-F^{2}\right)=0$

and

$Y_{i}^{\prime \prime}+S_{i \infty} F Y_{i}^{\prime}=0$

Figure 3 shows that, at values of $Y_{\mathrm{H}_{2}-\infty}$ of interest, these Fick's-law approximations yield concentration gradients that are much too small in the mass coordinate $\eta$, partially because of variable $C$ and $S_{i}$ 's and partially because of variable $\rho$. For constant density, $F^{\prime}=$ 1 in addition, which fails to reproduce the strong strain-rate variations found in undiluted systems [32]. If conditions in the fuel stream, rather than in the air stream, had been employed in the formulation for purposes of nondimensionalization, then the profiles in the standard approximations would be too steep, equally unsatisfactory, except at high fuelstream dilutions. It is concluded that molecular-weight and transport-property variations in isothermal hydrogen-air mixing layers necessitate taking into account property variations.

Although the Stefan-Maxwell description given in Eq. 14 is utilized here for the transport of oxygen and radicals, it is worth pointing out that non-Fickian effects, i.e., departures from a proportionality between $Y_{i} V_{i}$ and $Y_{i}^{\prime}$, are not very significant for major species in this flow, so that the diffusion velocity of $\mathrm{O}_{2}$ is also well reproduced by Fick's law if an adequately variable diffusion coefficient is employed. Neglecting the term proportional to $Y_{\mathrm{H}_{2}}^{\prime}$ simplifies Eq. 14 to

$\bar{\rho} Y_{i} \bar{V}_{i}=-C Y_{i}^{\prime} / S_{i}^{*}$,

where $S_{i}^{*}=\rho /\left(\mu D_{i}^{*}\right)$ is an appropriate Schmidt number based on the composition- 
weighted diffusion coefficient

$$
\begin{aligned}
D_{i}^{*}= & \left(1-Y_{\mathrm{H}_{2}}+\omega Y_{\mathrm{H}_{2}}\right) \\
& \times\left(\frac{1-Y_{\mathrm{H}_{2}}}{D_{i \mathrm{~N}_{2}}}+\frac{\omega Y_{\mathrm{H}_{2}}}{D_{i \mathrm{H}_{2}}}\right)^{-1}
\end{aligned}
$$

The accuracy of this Fickian transport description is illustrated in Fig. 3, where it can be seen that the resultant profile of $\mathrm{O}_{2}$ concentration follows closely that obtained with StefanMaxwell transport. It was found from similar comparisons that Eq. 33 also provides an accurate description for the transport of $\mathrm{O}$ atoms, while non-Fickian effects are quite important for $H$. It was seen that use of Eq. 33 results in large inaccuracies in the profiles of $\mathrm{H}$ concentration, which leads to underpredictions in the values of $\Delta_{c}$ by as much as $20 \%$ for undiluted configurations. Therefore, Stefan-Maxwell transport must be considered for $\mathbf{H}$ to obtain accurate results, while the Fickian description given in Eqs. 33 and 34 is sufficiently accurate for the diffusion velocities of $\mathrm{O}_{2}$ and $\mathrm{O}$. Nevertheless, since inclusion of non-Fickian effects does not complicate significantly the numerical integration of the bifurcation problem, Eq. 14 is employed here, not only for the diffusion velocity of $\mathrm{H}$, but also for those of $\mathrm{O}_{2}$ and $\mathrm{O}$.

\section{Radical Profiles}

Once the frozen field is determined, integration of Eqs. 25 and 26 provides the value of $\Delta_{c}$ and the eigenfunctions $y_{\mathrm{H}}$ and $y_{\mathrm{O}}$. Although these eigenfunctions do not possess information about the actual magnitude of the radical concentrations, they reproduce their profile shapes quite well when the variable molecular weight and transport are taken into account. This is shown in Fig. 4, where normalized eigenfunctions $y_{\mathrm{H}}^{*}=y_{\mathrm{H}} / y_{\mathrm{H} \max }$ and $y_{\mathrm{O}}^{*}=$ $y_{0} / y_{O \max }$ are compared with normalized radical profiles $y_{\mathrm{H}}^{*}=Y_{\mathrm{H}} / Y_{\mathrm{H} \max }$ and $y_{\mathrm{O}}^{*}=$ $Y_{O} / Y_{O \max }$ obtained from the numerical results at the critical point for $T_{\infty}=1400$. Corresponding graphs with the two standard models just identified gave much poorer agreement, especially for $Y_{\mathrm{H}_{2-x}}=0.5$, as a consequence of the poor inert-mixing description; these models are not considered further here.

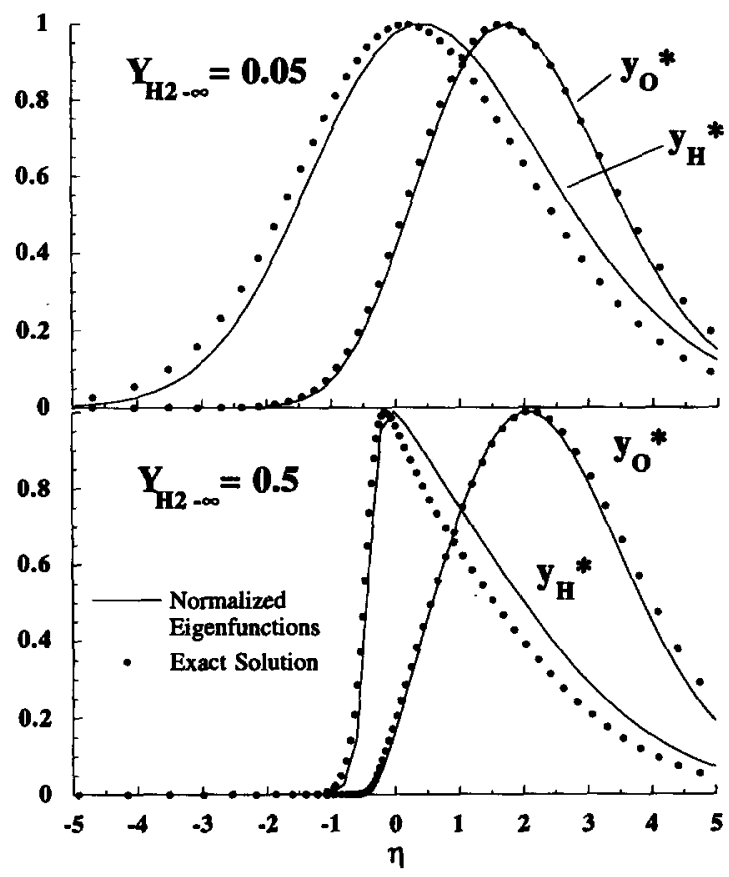

Fig. 4. A comparison for $Y_{\mathrm{H}_{2}-\infty}=0.05$ and $Y_{\mathrm{H}_{2}-\infty}=0.5$ of the normalized radical profiles obtained from numerical integration of the full equations at ignition with the normalized eigenfunctions obtained from integration of Eqs. 25 and 26 at atmospheric pressure and $T_{\infty}=1400 \mathrm{~K}$.

The results from Eqs. 25 and 26 also provide the ratio $y_{\mathrm{O} \max } / y_{\mathrm{H}_{\max }}$, which can be used to determine the ratio of maximum radical concentrations at ignition through

$$
\frac{Y_{\mathrm{O} \max }}{Y_{\mathrm{H} \max }}=\frac{Y_{\mathrm{O}_{2} \infty}}{2 Y_{\mathrm{H}_{2}-\infty}} \frac{y_{\mathrm{O} \max }}{y_{\mathrm{H} \max }} ;
$$

these results agree with the full numerical results quite well, although Eq. 35 somewhat overpredicts the ratio $Y_{\mathrm{O} \text { max }} / Y_{\mathrm{H} \text { max }}$, with overpredictions ranging between $15 \%$ and $35 \%$ as the fuel concentration increases from $Y_{\mathrm{H}_{2}-\infty}$ $=0.05$ to $Y_{\mathrm{H}_{2}-\infty}=1.0$. These inaccuracies are mainly attributable to the reduced chemical mechanism employed in the bifurcation analysis and correlate fairly well with the corresponding inaccuracies found in the predictions of critical conditions, which will be shown below to give strain rates at ignition between $15 \%$ and $25 \%$ higher than those obtained from numerical integrations of the conservation equations with detailed chemistry over the same dilution range. 


\section{Critical Strain Rate}

As mentioned before, integration of Eqs. 25 and 26 with the given boundary and nonnegativity conditions for $y_{H}$ and $y_{O}$ is possible only for a single value of $\Delta_{c}$ that is obtained as part of the solution. Use can then be made of the equation $\Delta_{c}=\left(\rho_{x} Y_{\mathrm{O}_{2}{ }^{x}} k_{1 \times}\right) /\left(W_{\mathrm{O}_{2}} a_{x}\right)$ to determine the value of the critical strain rate for a given temperature, pressure and fuel-stream dilution. Although we saw in Ref. 7 that for temperatures close to crossover the value of $J_{c}$ is strongly dependent on $T_{x}$, so that $\Delta_{c} \rightarrow \infty$ as $T_{x} \rightarrow T_{c}$, this dependence is rather weak at higher temperatures, for which $\gamma \ll 1$. It was found that the single curve of $\Delta_{c}$ as a function of $Y_{\mathrm{H}_{2}-x}$, given in Fig. 5, suffices to determine the critical value of $a_{x}$ for temperatures such that $T_{x}-T_{c} \gtrsim 150 \mathrm{~K}$. Critical strain rates computed from this curve at $p=1$ atm are compared with numerical results in Fig. 5,

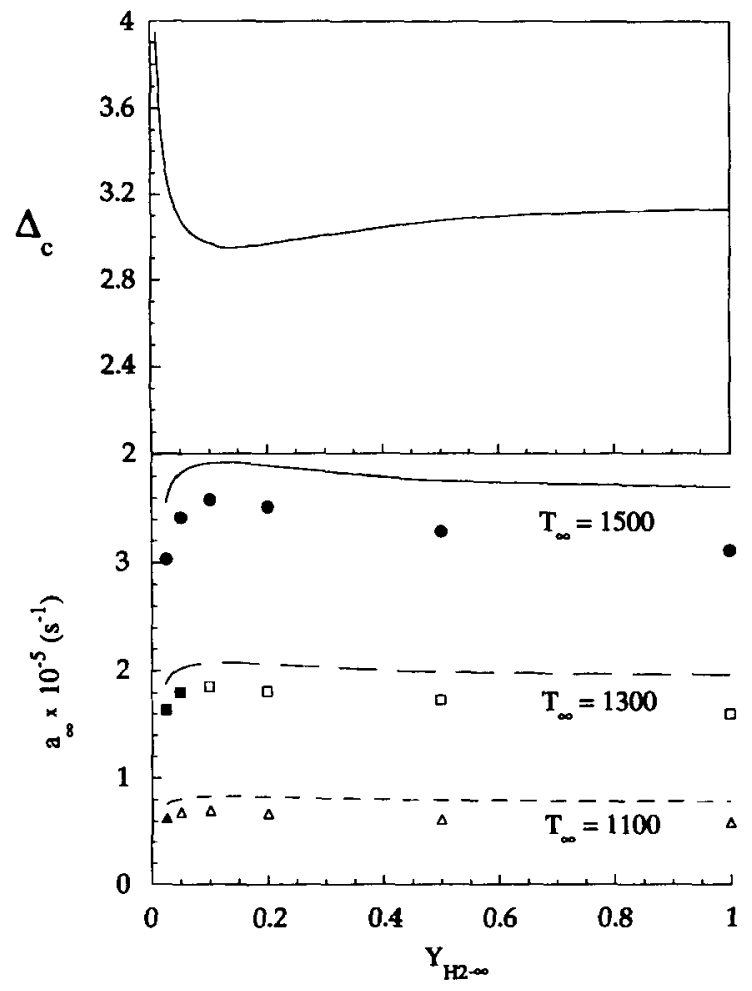

Fig. 5. The dependence of the critical strain rate on $Y_{\mathrm{H}_{2}-\infty}$ obtained from bifurcation analysis (lines) and from numerical integrations (symbols); the bifurcation-analysis results are computed from the $\Delta_{c}$ curve shown at the top of the figure. where empty symbols denote abrupt ignition behaviors $\left(T_{x}<T_{s}\right)$ and solid symbols correspond to smooth transitions $\left(T_{x}>T_{s}\right)$. The curves lie slightly above the points, mainly because of the three-step chemistry approximation, as explained below. It is remarkable that, except in highly diluted systems, the critical strain rate is independent of the degree of dilution of the fuel stream, a result that was also obtained in Ref. 7. In the previous investigation this independence was a direct consequence of the flowfield model, with constant density and strain rate, and the two-step chemistry employed, with rates independent of molecular hydrogen concentration, but in the present calculations it is due to cancelation of different effects, as explained below.

\section{EXPLANATIONS OF THE RESULTS OF THE COMPARISONS}

To better understand the interactions between flow and chemistry in the ignition process, a summary of the results of different approaches to the problem at $p=1 \mathrm{~atm}$ and $T_{\infty}=1400 \mathrm{~K}$ is given in Fig. 6 , where lines are the results from the linear bifurcation analyses for different flowfield models and different kinetic mechanisms, while symbols correspond to numerical integration of the complete differential equations with detailed chemistry and with the three-step reduced chemistry.

At this temperature and pressure the numerical analysis indicates that no abrupt ignition occurs, so that the form of the ignited curve is that shown in Fig. 1b irrespective of the degree of dilution. Before a detailed analysis of the different curves given in Fig. 6 is attempted, it is important to recall that, as explained in the previous investigation [7], the loss of stability of the frozen solution is associated with a certain critical value of the ratio of the characteristic residence time to the characteristic chemical time for production of radicals $\left(t_{\mathrm{FLOW}} / t_{\mathrm{CHEM}}\right)_{c}$. The interactions between the flow field and the chemistry can be quantitatively explained by the principle that this ratio remains approximately constant at ignition. Thus, if for instance one alters the ignition conditions by enhancing the chemical reactions, the corresponding critical strain rate 
will become larger, so that the ratio $\left(t_{\mathrm{FLOW}} / t_{\mathrm{CHEM}}\right)_{c}$ remains unchanged.

\section{The Two-Step Chemistry Description}

As can be seen from the graph, bifurcation analyses corresponding to two-step reduced chemistry, with $\mathrm{O}, \mathrm{OH}$, and $\mathrm{HO}_{2}$ in steady state, clearly overpredict the critical strain rate. To explain this, one must realize that by putting a certain species in steady state we are effectively reducing the characteristic production time corresponding to the remaining species. Therefore, for the same ratio $\left(t_{\mathrm{FLOW}} / t_{\mathrm{CHEM}}\right)_{c}$, the more species we put in steady state, the smaller the characteristic flow time at the critical point is. This not only explains why the two-step mechanism clearly overpredicts the strain rate at the critical point but also clarifies the results shown in Fig. 5, where the curves corresponding to bifurcation analysis, with species $\mathrm{OH}$ and $\mathrm{HO}_{2}$ in steady state, always stand above the numerical results with 21-step

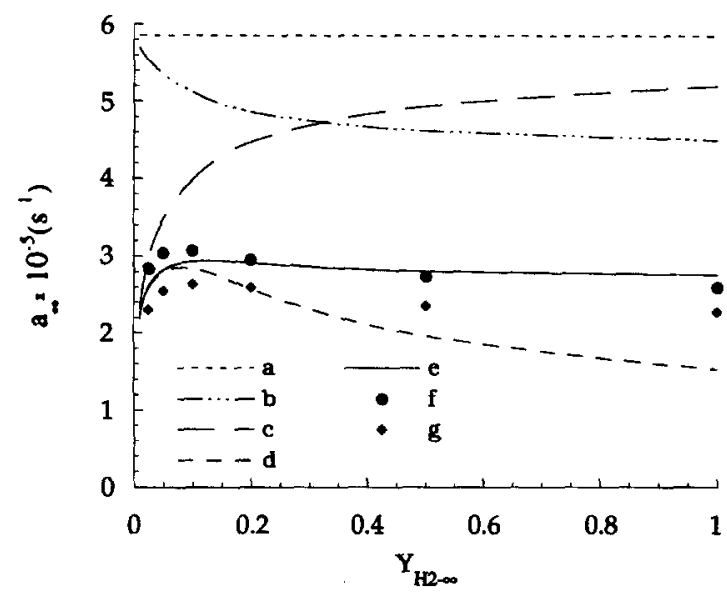

Fig. 6. The variation of the critical value of $a_{\infty}$ with $Y_{\mathrm{H}_{2}-\infty}$ at $T_{\infty}=1400 \mathrm{~K}$ and $p=1$ atom as obtained from the bifurcation analysis (curves) and from numerical integrations (points); (a) constant density and two-step reduced chemistry, (b) proposed simplified flowfield and two-step reduced chemistry, (c) same as (a) except with three-step reduced chemistry, (d) variable density but a ChapmanRubesin parameter of unity with three-step reduced chemistry, (e) same as (b) but with three-step chemistry, (f) numerical integrations with three-step reduced chemistry and (g) numerical integrations with the 21-step mechanism. chemistry. This clearly suggests that relaxing the steady state for $\mathrm{OH}$ would further improve the accuracy of the bifurcation analysis, but such an improvement was not attempted because the resulting changes would not be very large.

The curves obtained from the bifurcation analysis with two-step chemistry allow us to study separately the effect of the presence of a light species in one of the feed streams. It can be seen that increasing the concentration of $\mathrm{H}_{2}$ causes the critical strain rate to decrease when the constant-density model is replaced by the present model. This decrease is attributable mostly to the variation in characteristic flow time that occurs with variable density. As $Y_{\mathrm{H}_{2}-\infty}$ increases, the fuel stream becomes lighter, and conservation of momentum in the transverse direction requires that it must also achieve a higher strain rate according to the relation $\left(a_{-\infty} / a_{\infty}\right)=\sqrt{\rho_{\infty} / \rho_{-\infty}}$, resulting in a lower effective average residence time for the same value of $a_{\infty}$. Since no variation of chemical time occurs in this case, critical conditions are associated with a constant value of $t_{\text {FLOW }}$, which must then be achieved by decreasing $a_{\infty}$ with increasing $Y_{\mathrm{H}_{2}-\infty}$.

It can also be noticed that the analysis corresponding to two-step chemistry and variable density becomes more accurate for larger values of $Y_{\mathrm{H}_{2}-\infty}$, mainly because as $Y_{\mathrm{H}_{2}-\infty}$ increases the $\mathrm{O}$ atoms maintain steady state over a wider part of the mixing layer; however, the two-step results still exhibit substantial errors even at $Y_{\mathrm{H}_{2}-\infty}=1$. To explain why the improvement in agreement is not greater, one must investigate further the structure of the radical-pool profiles. The analysis is presented in an appendix. Although we adopt the constant-density approximation for simplicity there, the asymptotic structure that is identified in independent of the flowfield approximations and, therefore, the conclusions drawn are of general applicability.

It is shown in the appendix that the flowfield at ignition can be divided into three distinct layers as indicated in Fig. 7: a left outer layer where $O$ atoms maintain steady state, a right outer layer where the concentration of $\mathrm{H}_{2}$ is negligibly small and a thin layer where reaction 2 freezes the transition between the outer lay- 


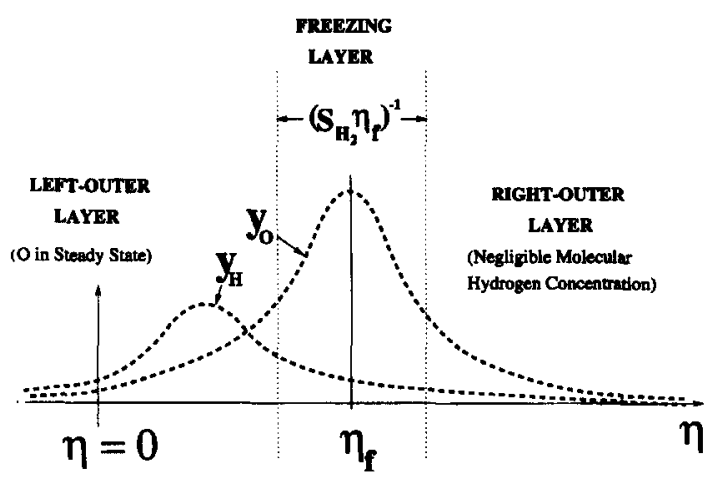

Fig. 7. A sketch of the radical pool structure at ignition.

ers takes place. It is seen that in the limit $s \rightarrow \infty$ the steady-state assumption for $\mathrm{O}$ holds cverywhere, and the zeroth-order solution that is obtained for $\Delta_{c}$ reduces to that of Ref. 7, with the exponential decay requirement for the eigenfunction $y_{\mathrm{H}}$ emerging naturally as a consequence of the matching of the solutions in the different layers. It is also seen that the first correction to the value of $\Delta_{c}$ is of order

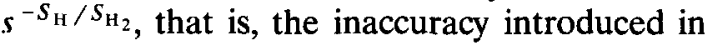
the prediction of $\Delta_{c}$ when the steady-state assumption for $\mathrm{O}$ is adopted everywhere is proportional to $s^{-S_{\mathrm{H}} / S_{\mathrm{H}_{2}}}$. Since the diffusivity of $\mathrm{H}$ atoms is considerably larger than that of molecular hydrogen, this quantity remains relatively large even for undiluted fuel feed, drastically limiting the accuracy of the two-step chemical-kinetic mechanism. Hence we can conclude that, although qualitative behaviors of nonpremixed $\mathrm{H}_{2}-\mathrm{O}_{2}$ ignition are adequately described with a single chain-branching radical, as was done in Ref. 7, one must account for non-steady-state $\mathrm{O}$-atom branching if reasonably accurate results are to be obtained.

\section{Dependence of the Solution on the Flowfield Description}

For the bifurcation results with three-step chemistry shown in Fig. 6, three different flowfield models were employed, the approximate description proposed herein and the two Fick's-law models previously discussed. As expected, the three analyses agree for highly diluted flames $Y_{\mathrm{H}_{2}-\infty} \leq 0.1$, for which density is nearly constant, and all three models yield similar flowfield descriptions. Since the rate corresponding to step II is proportional to the concentration of molecular hydrogen, increasing $Y_{\mathrm{H}_{2}-\infty}$ effectively reduces the chemical time of radical production, thereby increasing the strain rate at extinction, an effect exhibited by all three curves at small values of $Y_{\mathrm{H}_{2}-\infty}$. At larger values of $Y_{\mathrm{H}_{2}-\infty}$ this effect is reversed in all results except those of the constant-density analysis, which fails to reproduce the fluid-mechanical reduction in the average residence time that occurs as hydrogen is added to the fuel stream. In the two flowfield models that account for variable strain rate, whether the critical $a_{\infty}$ increases or decreases with increasing $Y_{\mathbf{H}_{2}-\infty}$ depends on the competition of reductions in the two characteristic times. The numerical results indicate that the two effects cancel each other almost exactly for $Y_{\mathrm{H}_{2}-\infty} \geq$ 0.1 , so that no variation in the critical $a_{\infty}$ is observed. This behavior is well reproduced by the proposed flowfield model, while the variable-density model with $C=1$ underpredicts the value of the critical strain rate for highly undiluted cases by overestimating the magnitude of the flow-time variation.

The full numerical integrations for three-step chemistry are seen in Fig. 6 to be in excellent agreement with the results of the bifurcation analysis with the proposed flowfield simplifications for three-step chemistry, over the entire range of $Y_{\mathrm{H}_{2}-\infty}$. This shows that the proposed flowfield simplifications are quite accurate. On the other hand, the numerical integrations with the 21-step chemistry are seen to yield ignition values for $a_{\infty}$ that are between $15 \%$ and $25 \%$ lower. This suggests that the four-step chemistry that is obtained by relaxing the steadystate assumption for $\mathrm{OH}$ is needed for obtaining truly accurate results as has been concluded earlier [5].

\section{INFLUENCE OF THE BOUNDARY TEMPERATURES ON IGNITION CONDITIONS}

\section{Effect of Different Temperatures}

Since in most applications cold hydrogen is injected into a cold stream of air, consideration must be given to configurations with fuel-side temperatures below $T_{\infty}$. In Ref. 7 it was found 
that when $O$ is assumed to be in steady state, which results in no chemical activity taking place in the absence of $\mathrm{O}_{2}$, the ignition process is almost entirely independent of the temperature of the fuel stream. In the three-step approximation, however, the generation of $\mathrm{H}$ through reaction II occurs mainly on the hydrogen side of the mixing layer, and variations of $T_{-\infty}$ are then expected to have a larger influence on the process. To determine the extent of this influence, the critical strain rates were calculated as functions of the nondimensional temperature difference $\theta_{-\infty}\left(T_{-\infty}-\right.$ $\left.T_{\infty}\right) / T_{\infty}$ for two different temperatures of the air stream and for a highly diluted fuel. For $Y_{\mathrm{H}_{2}-\infty} \ll 1$ the constant-density model gives a reasonably good description of the flow field, and Eqs. A4 and A5 can be employed to determine $\Delta_{c}$. The results are exhibited in Fig. 8. As expected, as one lowers $T_{-\infty}$ the value of the critical residence time increases to balance the corresponding increase in characteristic chemical time of radical production. However, since the value of $\beta_{2}$ is relatively small, the dependence of critical conditions on $T_{-\infty}$ is still quite weak. Results from numerical integration of the full equations with detailed chemistry are also included in Fig. 8. The agreement between the bifurcation and numerical predictions is reasonably good, the difference being attributable to the inaccuracy of the three-step chemistry, as may be inferred from Fig. 6.

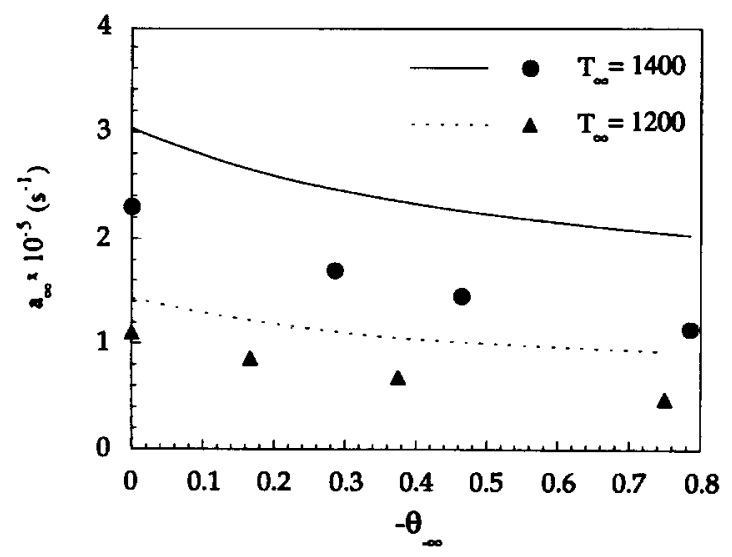

Fig. 8. The effect of variation in the fuel temperature $T_{-\infty}=T_{\infty}\left(1+\theta_{-\infty}\right)$ on the ignition strain rate at atmospheric pressure for $Y_{\mathrm{H}_{2}-\infty}=0.025$ and two different values of $T_{\infty}$, as obtained from the bifurcation analysis (lines) and numerical integrations (symbols).
Similar agreement would be expected at larger values of $Y_{\mathrm{H}_{2}-\infty}$ if the proposed simplified flow model accounting for changes in density and transport properties is used in the bifurcation analysis.

\section{Criterion for Existence of a Chain-Branching Explosion}

Inclusion of a second chain-branching radical does not modify previous results concerning the conditions that are necessary for existence of a bifurcation phenomenon. The qualitative analysis presented in Ref. 7 indicated that a chain-branching explosion is possible only for temperatures of the oxidizer stream above crossover $(\gamma<1)$, for which the net production of $\mathrm{H}$ is positive. At the crossover temperature, the rates of consumption and production of $\mathrm{H}$ atoms become equal, the characteristic chemical time for production of $\mathrm{H}$ becomes infinite, and a zero strain rate is required for ignition to occur. The same criterion applies when the steady-state assumption is relaxed for $\mathrm{O}$, that is, Eqs. 25 and 26 have solutions only for values of $\gamma$ below unity, so that production of both radicals can take place simultaneously over some extent of the mixing layer. This easily can be illustrated by manipulation of the radical conservation equations of the constantdensity flowfield model. Combining linearly Eqs. A4 and A5 and integrating the resultant expression between $\eta=-\infty$ and $\eta=\infty$ yields

$$
\begin{aligned}
& \int_{-\infty}^{\infty}\left(s y_{\mathrm{H}}+y_{\mathrm{O}}\right) d \eta \\
& \quad=s \Delta_{c} \int_{-\infty}^{\infty}\left[\exp \left(\beta_{1} \theta\right)-\gamma\right] y_{\mathrm{O}_{2}} y_{\mathrm{H}} d \eta .
\end{aligned}
$$

In deriving this equation, integration by parts was employed to generate the final expression. Since $\theta_{-\infty} \leq 0$ in practical applications and for all conditions considered here, it follows that $\theta \leq 0$ and that therefore Eq. 36 can be satisfied with positive radical concentrations only if $\gamma<1$. If $\theta_{-\infty}$ were positive, then it would be appropriate to redefine $\gamma$ in terms of conditions at $-\infty$, instead of at $\infty$, and the conclusion would continue to hold. In general, when- 
ever the formulation yields a problem of the bifurcation type, the bifurcation will cease to exist when the maximum temperature is below $T_{c}$, and a thermal-explosion type of analysis for ignition will then be needed instead. For example, the conclusion may be expected to apply also to four-step chemistry, which emerges by relaxing the steady-state assumption for $\mathrm{OH}$.

\section{First and Second Ignition Limits}

It has been found recently in a numerical study [3] that the ignition-limit curve of hydrogen-air counterflow diffusion flames, which gives the limiting boundary between ignitable and nonignitable states in a diagram of pressure as a function of the outer temperature, exhibits a $Z$-shape curve analogous to the explosion-limit curve of homogeneous $\mathrm{H}_{2}-\mathrm{O}_{2}$ mixtures. While the third ignition limit correspond to highpressure conditions involving thermal explosions, a regime not considered here, the first and second ignition limits, which form the lower peninsula of the Z-shape curve, are explained by the present analysis and can be determined from the equation

$p=\frac{R^{o} T_{\infty} a_{\infty}}{Y_{\mathrm{O}_{2}} k_{1 \infty}} \Delta_{c}$,

obtained from the definition of the critical Damköhler number. In this equation, $\Delta_{c}$ is evaluated for a given pressure and temperature by integration of Eqs. 25 and 26. A simple computation of the resultant $\mathrm{C}$-shape curves can be performed by considering separately the first and second ignition limits as follows.

As mentioned before, for temperatures sufficiently above crossover the rate of the recombination reaction is very small, and the ignition process is determined by the competition between radical branching and radical loss, the rate of which is proportional to the strain rate. In this case $(\gamma \ll 1)$ the value of $\Delta_{c}$ is a constant for a given fuel feed dilution as shown in Fig. 5. Hence, using this figure together with Eq. 37 the limiting pressure as a function of $T_{\infty}$ corresponding to the first ignition limit can be casily computed for a given strain rate and dilution. The resulting limiting pressure increases as the temperature decreases, as can be seen from Fig. 9, where limit curves corresponding to five different strain rates and undiluted fuel feed are presented.

The value of $\Delta_{c}$ ceases being a constant as the temperature approaches crossover. As $\gamma$ $\rightarrow 1$ the value of $\Delta_{c}$ diverges towards infinity with an asymptotic growth given in a first approximation by $\Delta_{c} \propto(1-\gamma)^{-1}$, a result that was previously obtained [7] with two-step reduced chemistry and that also hold for threestep chemistry, as can be seen from Eq. 36. The limit curve then turns around and begins to describe the upper branch of the C-shape curve, rapidly approaching the curve $T_{\infty}=T_{c}$. Although a detailed description of the turning point requires a careful evaluation of Eq. 37 near the crossover temperature, for practical purposes one can use the approximate limit curve that is obtained by extending the lower branch, determined as explained above, to the point at which the crossover temperature is reached, and then replacing the upper branch with the curve $T_{\infty}=T_{c}$, which is valid away from the turning point. The resulting simplified curves are shown in Fig. 9, where the upper branch, corresponding to the second explosion limit, is determined from the equation $y=1$.

\section{CONCLUSIONS}

We have seen that bifurcation methods can be applied to the analysis of high-temperature

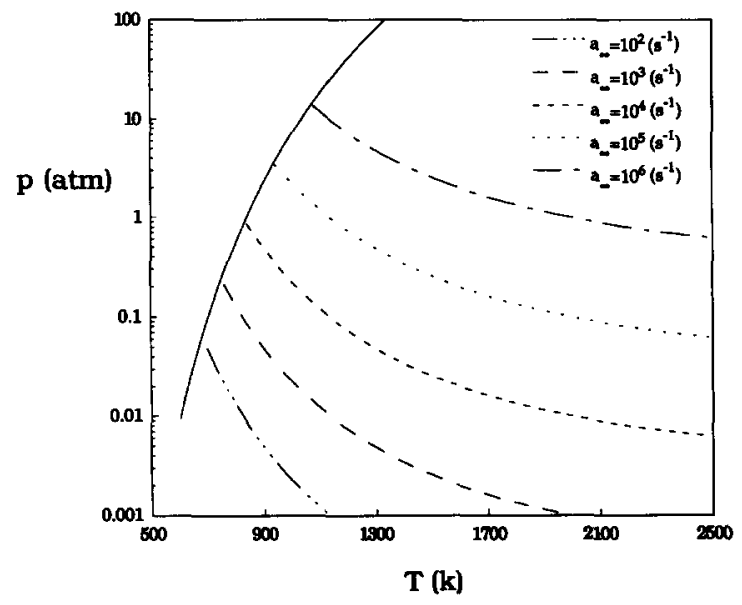

Fig. 9. First-ignition-limit curves as obtained from Eq. 37 with $\Delta_{c}=3.1$ and second-ignition-limit curve (solid line) as obtained from $\gamma=1$. 
ignition in nonpremixed hydrogen-oxygen environments, not only to describe the process qualitatively, but also to make accurate predictions regarding critical conditions for ignition. Applicability of a similar analysis to the study of high-temperature nonpremixed autoignition of other fuels, for example hydrocarbons such as methane, and with different geometries, for example spherical droplet autoignition, can be anticipated.

By investigating the structure of the radical pool for fuel-rich systems, for which the oxygen-to-fuel mass ratio, $s$, is a large quantity, it was found that adoption of the steady-state assumption for $\mathrm{O}$ everywhere in the flow field causes inaccuracies in predicting critical Damköhler numbers for ignition that are of order $s^{-S_{\mathrm{H}} / s_{\mathrm{H}_{2}}}$, a fairly large quantity for all dilution conditions. This result is in agreement with results of previous numerical studies [5, 14] and clearly indicates that, although a simple two-step kinetic mechanism suffices to describe the qualitative aspects of the nonhomogeneous ignition process, as previously done [7], consideration of a three-step mechanism, with $\mathrm{H}$ and $\mathrm{O}$ as the two chain-branching species, is essential to provide accurate predictions of critical ignition conditions. Because of the particular nature of the fuel under study, the accuracy of the bifurcation results obtained with a constant-density flowfield model and a Fickian law with constant Schmidt numbers for diffusion is restricted to highly diluted fuels. Hence, a simplified description for the $\mathrm{H}_{2}-$ $\mathrm{O}_{2}-\mathrm{N}_{2}$ mixing layer with Stefan-Maxwell transport and variable density, strain rate and transport properties was implemented here in the bifurcation analysis. Results obtained with this model and with a three-step description of the reduced chemistry were shown to be in excellent agreement with those obtained by numerical integration of the full conservation equations with detailed chemistry over a wide range of temperatures and dilutions.

This work was supported by the National Science Foundation through Grant No. CTS-9214888. The work of A. Liñan was partially supported by the Spanish CICYT under Contract No. P1394-0400 and by INTA under Contract No. INTA 4070-0036/1995. ALS wants to thank
Prof. P. L. García-Ybarra and Dr. J. S. Kim for interesting discussions on the subject and $G B$ would like to thank Prof. M. D. Smooke for providing the numerical code used in this study.

\section{APPENDIX: THE ASYMPTOTIC STRUCTURE OF THE RADICAL POOL AT IGNITION}

As mentioned in the main text, we shall adopt for simplicity the constant-density model to describe the flow field. The solution for the stream function then reduces to $F=\eta$, while the frozen temp:rature and reactant concentration are given by

$$
\begin{aligned}
& \theta=\frac{\theta_{-\infty}}{2} \operatorname{erfc}\left(\sqrt{\frac{\operatorname{Pr}}{2}} \eta\right), \\
& y_{\mathrm{O}_{2}}=1-\frac{1}{2} \operatorname{erfc}\left(\sqrt{\frac{S_{\mathrm{O}_{2}}}{2} \eta}\right)
\end{aligned}
$$

and

$y_{\mathrm{H}_{2}}=\frac{1}{2} \operatorname{erfc}\left(\sqrt{\frac{S_{\mathrm{H}_{2}}}{2}} \eta\right)$.

The first-order equations for the radical concentrations then reduce to

$$
\begin{aligned}
y_{\mathrm{H}}^{\prime \prime}+ & S_{\mathrm{H}} \eta y_{\mathrm{H}}^{\prime} \\
& =-S_{\mathrm{H}} \Delta_{c}\left[r \exp \left(\beta_{2} \theta\right) y_{\mathrm{H}_{2}} y_{\mathrm{O}}-\gamma y_{\mathrm{O}_{2}} y_{\mathrm{H}}\right]
\end{aligned}
$$

and

$$
\begin{aligned}
y_{\mathrm{O}}^{\prime \prime}+S_{\mathrm{O}} \eta y_{\mathrm{O}}^{\prime}= & -S_{\mathrm{O}} s \Delta_{c}\left[\exp \left(\beta_{1} \theta\right) y_{\mathrm{O}_{2}} y_{\mathrm{H}}\right. \\
& \left.-r \exp \left(\beta_{2} \theta\right) y_{\mathrm{H}_{2}} y_{\mathrm{O}}\right],
\end{aligned}
$$

with boundary conditions $y_{\mathrm{H}}( \pm \infty)=0$ and $y_{0}( \pm \infty)=0$. Any nonexponential dependence of the reaction-rate constants on temperature is neglected. Typical values of $\beta_{i}=E_{i} /\left(R^{o} T_{\infty}\right)$ are $\beta_{1}=7$ and $\beta_{2}=3$, when all temperature dependences are absorbed into the Arrhenius exponential.

For fuel-rich configurations the parameter $s$ takes on very large values, and it is therefore appropriate to consider the limit $s \rightarrow \infty$ to study the asymptotic solution to Eqs. A4 and A5. In this limit Eq. A5 yields $\exp \left(\beta_{1} \theta\right) y_{\mathrm{O}_{2}} y_{\mathrm{H}}$ 
$=r \exp \left(\beta_{2} \theta\right) y_{\mathrm{H}_{2}} y_{\mathrm{O}}$, which corresponds to the steady state for $\mathrm{O}$ atoms. This result holds on the fuel side of the mixing layer and also far into the oxidizer side, up to a point, $\eta=\eta_{f} \rightarrow 1$, where the concentration of $\mathrm{H}_{2}$ is so small that the chemical terms in Eq. A5 becomes of the order of the transport terms and the steadystate assumption breaks down. Farther into the oxidizer stream, the concentration of molecular hydrogen is negligible, and the chemical activity reduces to that associated with steps 1 and 3 , whose rates are independent of $\mathrm{H}_{2}$. Overall, the mixing layer is composed of three distinct regions that are sketched in Fig. 7, namely, a left outer layer where $O$ atoms are in steady state, a right outer layer where reaction 2 is frozen because of the absence of $\mathrm{H}_{2}$, and a thin freezing layer of characteristic thickness $\delta_{f}$ where the transition between the outer layers takes place and where $y_{0}$ evolves from the growing steady-state solution to a decaying solution. The thickness $\delta_{f}$, infinitesimally small in the limit $s \rightarrow \infty$, is associated with the $\mathrm{H}_{2}$ depletion process, since that is the key factor which determines the freezing of step 2 and, consequently, the departure of the $\mathrm{O}$ profile from the steady-state solution. Writing $y_{\mathrm{H}_{2}}$ in terms of the stretched variable $\imath=\left(\eta-\eta_{f}\right) / \delta_{f}$, used later for the description of the freezing layer, indicates that the appropriate choice of $\delta_{f}$ is $\left(S_{\mathrm{H}_{2}} \eta_{f}\right)^{-1}$, so that Eq. A5 will exhibit significant variations of $y_{\mathrm{H}_{2}}$ in this layer. Furthermore, use of this expression together with $\mathrm{Eq}$. A5 indicates that the value of $\eta_{f}$ must be such that $s y_{\mathrm{H}_{f}} / \eta_{f}^{2} \simeq 1$, where $y_{\mathrm{H}_{2}} \simeq \exp \left(-S_{\mathrm{H}_{2}} \eta_{f}^{2} / 2\right) /\left(2 \pi S_{\mathrm{H}_{2}}\right)^{1 / 2}$ is the value of $y_{\mathrm{H}_{2}}$ at $\eta=\eta_{f}$. Observation of the above relationship reveals that $\delta_{f}^{2}$ is of the order of $\ln (s)^{-1}$ in the first approximation. A convenient expression to determine $\eta_{f}$ is

$\frac{s r y_{\mathrm{H}_{2}}}{s_{\mathrm{O}} \eta_{f}^{2}} \Delta_{c 0}=1$,

with $\Delta_{c 0}$ being the limiting value of $\Delta_{c}$ for $s=\infty$.

The solution can be obtained by considering expansions of the eigenfunctions $y_{\mathrm{H}}$ and $y_{\mathrm{O}}$ and of the eigenvalue $\Delta_{c}$ in the small parameter $\delta_{f}^{2}$, so that, for instance, $\Delta_{c}=\Delta_{c 0}+\delta_{f}^{2} \Delta_{c 1}$ $+\cdots$. After solving for the radical concentrations in the different regions and matching, the resultant profiles determine $\Delta_{c}$.

Let the subscripts $l, f$, and $r$ identify the radical profiles in the left outer, freezing, and right outer layers, respectively. The equations corresponding to the left outer region can be readily shown from Eqs. A4 and A5 to be

$$
\begin{aligned}
y_{\mathrm{H}_{l}}^{\prime \prime}+ & S_{\mathrm{H}} \eta y_{\mathrm{H}_{l}}^{\prime} \\
& =-S_{\mathrm{H}} \Delta_{c 0}\left[\exp \left(\beta_{1} \theta\right)-\gamma\right] y_{\mathrm{O}_{2}} y_{\mathrm{H}_{l}}
\end{aligned}
$$

and

$y_{\mathrm{O}_{l}}=\frac{y_{\mathrm{O}_{2}} \exp \left[\left(\beta_{1}-\beta_{2}\right) \theta\right]}{y_{\mathrm{H}_{2}}} y_{\mathrm{H}_{l}}$,

where exponentially small terms of order $s^{-1}$ have been neglected. These equations determine the chain-branching radical concentrations on the fuel side and on part of the oxidizer side of the mixing layer. The $\mathrm{H}$ atoms are more diffusive than $\mathrm{H}_{2}$ molecules, which from Eq. A8 causes the value of $y_{\mathrm{O}_{l}}$ to increase as one moves into the oxidizer stream. Since the value of $\eta_{f}$ is very large and the diffusivities of heat and molecular oxygen are much smaller than those of $\mathrm{H}$ or $\mathrm{H}_{2}$, we can assume that $\theta=0$ and $y_{\mathrm{O}_{2}}=1$ when describing the freezing and right-outer regions. The zeroth-order equations that determine the $\mathrm{H}$ and $\mathrm{O}$ concentrations in the latter are then given by

$y_{\mathrm{H}_{r}}^{\prime \prime}+S_{\mathrm{H}_{\mathrm{H}}} \eta y_{\mathrm{H}_{r}}^{\prime}=S_{\mathrm{H}} \gamma \Delta_{c 0} y_{\mathrm{H}_{r}}$

and

$y_{\mathrm{O}_{r}}^{\prime \prime}+S_{\mathrm{O}} \eta y_{\mathrm{O}_{r}}^{\prime}=-S_{\mathrm{O}} s \Delta_{c 0} y_{\mathrm{H}_{r}}$,

whose solutions in first approximation are

$y_{\mathrm{H}_{r}}=\frac{\exp \left(-S_{\mathrm{H}} \eta^{2} / 2\right)}{\eta^{\Delta_{c 0} \gamma+1}}$

and

$y_{\mathrm{O}_{r}}=\frac{S_{\mathrm{O}} s \Delta_{c 0}}{S_{\mathrm{H}}\left(S_{\mathrm{O}}-S_{H}\right)} \frac{\exp \left(-S_{\mathrm{H}} \eta^{2} / 2\right)}{\eta^{\Delta_{c 0} \gamma+3}}$.

In obtaining the above solutions the boundary conditions at $\eta=\infty$ have been used, and 
an exponentially small term of the form $\exp \left(-S_{\mathrm{O}} \eta^{2} / 2\right) / \eta$ has been neglected in Eq. A12, since with $S_{\mathrm{H}}<S_{\mathrm{O}}$ this term is negligible in comparison with that which is retained. In addition the arbitrary amplitude of the solution has been chosen so that no constant multiplier appears on the right-hand side of Eq. Al1.

In the freezing layer it is convenient to define normalized functions $\bar{y}_{\mathrm{H}_{f}}$ and $\bar{y}_{\mathrm{O}_{f}}$ according to

$y_{\mathrm{H}_{f}}=\frac{\exp \left(-S_{\mathrm{H}} \eta_{f}^{2} / 2\right)}{\eta_{f}^{\Delta_{c 0} \gamma+1}} \bar{y}_{\mathrm{H}_{f}}$

and

$y_{\mathrm{O}_{f}}=\frac{\exp \left(-S_{\mathrm{H}} \eta_{f}^{2} / 2\right)}{r y_{\mathrm{H}_{f}} \eta_{f}^{\alpha_{c 0} \gamma+1}} \bar{y}_{\mathrm{O}_{f}}$.

From Eqs. A4 and A5, with use made of Eq. A6, the zeroth-order equations for $\bar{y}_{\mathrm{H}_{f}}$ and $\bar{y}_{\mathrm{O}_{f}}$ can be shown to be

$\frac{d^{2} \bar{y}_{\mathrm{H}_{f}}}{d v^{2}}+\frac{S_{\mathrm{H}}}{S_{\mathrm{H}_{2}}} \frac{d \bar{y}_{\mathrm{H}_{f}}}{d v}=0$

and

$\frac{d^{2} \bar{y}_{\mathrm{O}_{f}}}{d v^{2}}+\frac{S_{\mathrm{O}}}{S_{\mathrm{H}_{2}}} \frac{d \bar{y}_{\mathrm{O}_{f}}}{d v}=-\frac{S_{\mathrm{O}}{ }^{2}}{S_{\mathrm{H}_{2}}{ }^{2}}\left(\bar{y}_{\mathrm{H}_{f}}-e^{-v} \bar{y}_{\mathrm{O}_{f}}\right)$.

Integrating Eq. A15 and matching with $y_{\mathrm{H}_{r}}$ gives

$\bar{y}_{\mathrm{H}_{f}}=\exp \left(-\frac{S_{\mathrm{H}}}{S_{\mathrm{H}_{2}}} v\right)$.

We can now determine the value of $\Delta_{c 0}$ by matching $\bar{y}_{\mathbf{H}_{f}}$ with the solution of Eq. A7. For large values of $\eta$ the two solutions to this equation are given in first approximation by $\eta^{\left[\Delta_{c 0}(1-\gamma)-1\right]} \exp \left(-S_{\mathrm{H}} \eta^{2} / 2\right)$ and $\eta^{-\Delta_{c 0}(1-\gamma)}$. Matching with Eq. A17 rules out the algebraically decaying solution, so that $\Delta_{c 0}$ is simply determined as the eigenvalue for which there exists a nonnegative solution to Eq. A7 with exponential decay as $\eta \rightarrow \pm \infty$. It is of interest that in Ref. 7, where $\mathrm{O}$ was assumed to be in steady state, consideration of $\mathrm{H}_{2}$ depletion on the oxidizer stream led to the same result.

Finally, solving Eq. A16 determines the variation of the mass fraction of $\mathrm{O}$ atoms across the freezing layer. In order to find an analytical solution, it is convenient to introduce a new variable $u=\exp \left(-\left(S_{\mathrm{O}} / S_{\mathrm{H}_{2}}\right) v\right)$, which transforms Eq. A16 to

$$
\frac{d^{2}\left(\bar{y}_{\mathrm{O}_{f}}\right)}{d u^{2}}-u^{\left(s_{\mathrm{H}_{2}} / s_{\mathrm{O}}-2\right)} \bar{y}_{\mathrm{O}_{f}}=-u^{\left(s_{\mathrm{H}} / s_{\mathrm{O}}-2\right)} .
$$

Solving this equation and matching with the solutions from the two outer layers gives

$$
\begin{aligned}
\bar{y}_{\mathrm{O}_{f}}= & 2 \nu\left[u^{1 / 2} I_{\nu}\left(2 \nu u^{1 / 2 \nu}\right)\right. \\
& \times \int_{u}^{\infty} u^{\left(S_{\mathrm{H}} / s_{\mathrm{O}}-3 / 2\right)} K_{\nu}\left(2 \nu u^{1 / 2 \nu}\right) d u \\
& +u^{1 / 2} K_{\nu}\left(2 \nu u^{1 / 2 \nu}\right) \\
& \left.\times \int_{0}^{u} u^{\left(S_{\mathrm{H}} / s_{\mathrm{O}}-3 / 2\right)} I_{\nu}\left(2 \nu u^{1 / 2 \nu}\right) d u\right],
\end{aligned}
$$

where $\nu=S_{\mathrm{O}} / S_{\mathrm{H}_{2}}$ and $I_{\nu}$ and $K_{\nu}$ are modified Bessel functions of order $\nu$ [41]. The asymptotic behaviors of $\bar{y}_{\mathrm{O}_{f}}$ for large positive and negative values of $v$ are given by [41] $S_{\mathrm{O}}{ }^{2} \exp \left(-\left(S_{\mathrm{H}} / S_{\mathrm{H}_{2}}\right) v\right) /\left[S_{\mathrm{H}}\left(S_{\mathrm{O}}-S_{\mathrm{H}}\right)\right]$ and $\exp \left[\left(1-S_{\mathrm{H}} / S_{\mathrm{H}_{2}}\right) v\right]$, which correspond to the asymptotic expansions of the functions $y_{\mathrm{O}_{r}}$ and $y_{\mathrm{O}_{l}}$ as $\eta \rightarrow \eta_{f}{ }^{+}$and $\eta \rightarrow \eta_{f}{ }^{-}$, respectively. A plot of the $\bar{y}_{\mathrm{O}}$, profile in the freezing layer is shown in Fig. 10.

Extension of the solution to higher orders reveals that $\Delta_{c 1}=\Delta_{c 2}=\cdots=0$, i.e., corrections to the critical Damköhler number are exponentially small in $\delta_{f}^{2}$ and cannot be predicted by the algebraic expansion in $\delta_{f}^{2}$, which does lead to successive corrections to radical profiles. The analysis required for obtaining the first correction to $\Delta_{c 0}$ would involve consideration of an additional layer located at a distance of order $-\delta_{f} \ln \left(\delta_{f}^{2}\right)$ to the left of the freezing layer. In this region the concentration 


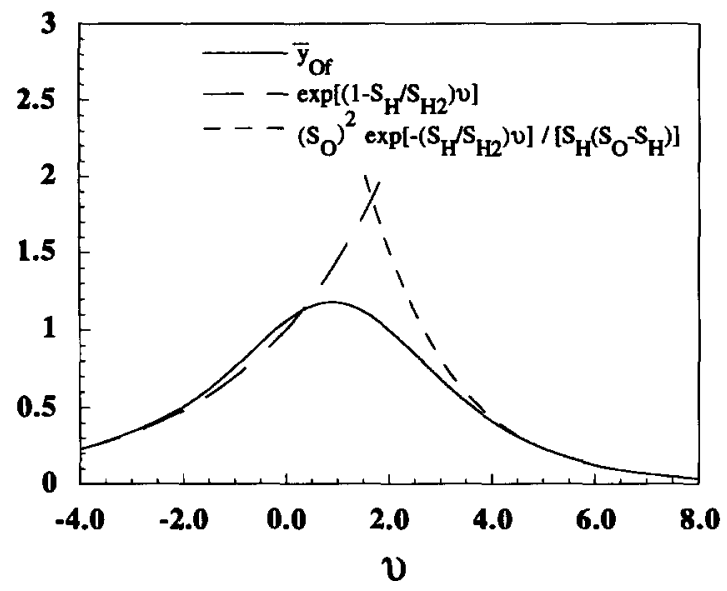

Fig. 10. The variation of $\bar{y}_{\mathrm{O}_{f}}$ across the freezing layer.

of $\mathrm{H}$ atoms, whose governing equation would contain both transport and chemical terms, evolves from a solution to Eq. A7 containing an algebraically decaying part, to the exponentially decaying profile given in Eq. A17. Although such an analysis, which would require expansions in terms of $\delta_{f}$ involving logarithmic terms, will not be considered here because of its anticipated very slow semiconvergence, the order of the first correction to the Damköhler number that would emerge can be easily anticipated as follows.

Combining Eqs. A4 and A5 yields

$$
\begin{aligned}
y_{\mathrm{H}}^{\prime \prime}+ & S_{\mathrm{H}} \eta y_{\mathrm{H}}^{\prime} \\
= & -S_{\mathrm{H}} \Delta_{c}\left[\exp \left(\beta_{1} \theta\right)-\gamma\right] y_{\mathrm{O}_{2}} y_{\mathrm{H}} \\
& -\frac{1}{s} \frac{S_{\mathrm{H}}}{S_{\mathrm{O}}}\left(y_{\mathrm{O}}^{\prime \prime}+S_{\mathrm{O}} \eta y_{\mathrm{O}}^{\prime}\right) .
\end{aligned}
$$

The right-hand side of this equation reveals that $\left(\Delta_{c}-\Delta_{c 0}\right)$ is of the order of $y_{\mathrm{O} \max } / s$. Also, from Eq. A14 it is seen that the order of the peak value of $y_{\mathrm{O}}$ in first approximation is $s^{1-s_{\mathrm{H}} / s_{\mathrm{H}_{2}}}$, yielding finally the result that $\left(\Delta_{c}-\right.$ $\left.\Delta_{c 0}\right)$ is of order $s^{-S_{\mathrm{H}} / s_{\mathrm{H}_{2}}}$. This prediction is tested in Fig. 11, which exhibits the variation of $\Delta_{c}$ with $s$, obtained numerically by integrating Eqs. A4 and A5 in the case $\theta_{-\infty}=0$ and $\gamma=0$. As can be seen, the critical Damköhler number approaches $\Delta_{c 0}=1.4726$ linearly in the appropriate power of $s$, as predicted by the above reasoning. This scaling law indicates that the inaccuracy introduced in the result by as-

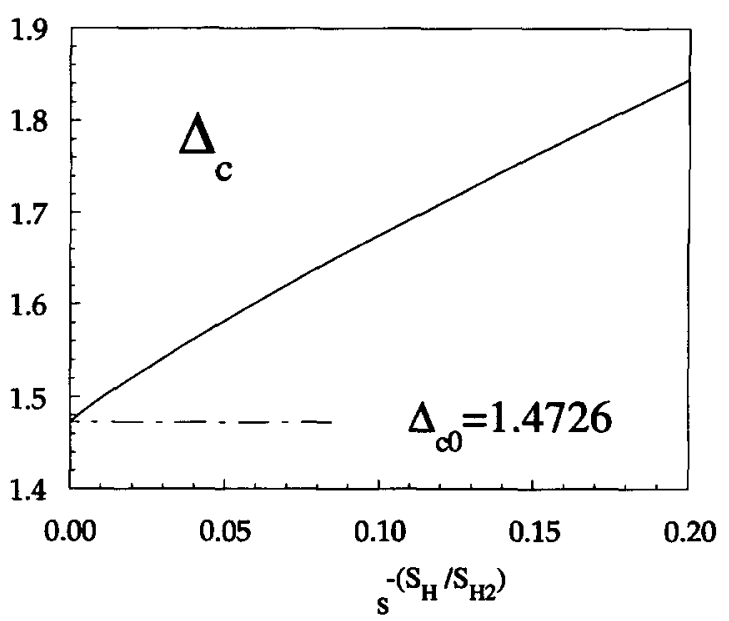

Fig. 11. The variation of the critical Damköhler number with $s$ for $\gamma=0$ and $r=1.5$ in the isothermal mixing layer.

suming $\mathrm{O}$ atoms to be in steady state is of $\operatorname{order} s^{-s_{\mathrm{H}} / s_{\mathrm{H}_{2}}}$.

\section{REFERENCES}

1. Balakrishnan, G., and Williams, F. A., J. Prop. Power 10:434-436 (1994).

2. Darabiha, N., and Candel, S., Combust. Sci. Technol. 86:67-85 (1992).

3. Kreutz, T. G., and Law, C. K., Combust. Flame 104:157-175 (1996).

4. Kreutz, T. G., Nishioka, M., and Law, C. K., Combust. Flame 99:758-766 (1994).

5. Balakrishnan, G., Smooke, M. D., and Williams, F. A., Combust. Flame 102:329-340 (1994).

6. Lee, S. R., and Law, C. K., Combust. Sci. Technol. 97:337-389 (1994).

7. Sánchez, A. L., Liñán, A., and Williams, F. A., Twenty-Fifth Symposium (International) on Combustion, The Combustion Institute, Pittsburgh, 1994, pp. 1529-1537.

8. Liñán, A., Acta Astronaut. 1:1007-1039 (1974).

9. Liñán, A., and Crespo, A., Combust. Sci. Technol. 14:95-117 (1976).

10. Treviño, C., Prog. Astronaut. Aeronaut. 131:19-43 (1991).

11. Niioka, T., Combust. Flame 76:143-149 (1989).

12. Ju, Y., and Niioka, T., ALAA J. 31:863-868 (1993).

13. Treviño, C., Méndez, F., Combust. Sci. Technol. 78: 197-216 (1991).

14. Ju, Y., and Niioka, T., Combust. Flame 99:240-246 (1994).

15. Treviño, C., and Liñán, A., Combust. Flame, 103:129-141 (1995).

16. Drazin, P. G., and Reid, W. H., Hydrodynamic Stability, Cambridge University Press, Cambridge, 1981, pp. 407-415. 
17. Vlachos, D. G., Schmidt, L. D., and Aris, R., Combust. Flame 95:313-335 (1993).

18. Sánchez, A. L., and García-Ybarra, P. L., in preparation.

19. Sen, A. K., and Ludford, G. S. S., Eighteenth Symposium (International) on Combustion, The Combustion Institute, Pittsburgh, 1981, pp. 417-422.

20. Chelliah, H. K., and Williams, F. A., Combust. Sci. Technol. 51:129-144 (1987).

21. García-Ybarra, P. L., and Treviño, C., Combust. Flame 96:293-303 (1994).

22. Da Silva, L. F. F., and Deshaies, B., Twenty-Fifth Symposium (International) on Combustion, The Combustion Institute, Pittsburgh, 1994, pp. 29-36.

23. Baulch, D. L., Cobos, C. J., Cox, R. A., Esser, C., Frank, P., Just, T. Kerr, J. A., Philling, M. J., Troe, J., Walker, R. W., and Warnatz, J., J. Phys. Chem. Ref. Data 21:411-736 (1992).

24. Smooke, M. D., Reduced Kinetic Mechanisms and Asymptotic Approximations for Methane-Air Flames, Springer-Verlag, New York, 1991.

25. Baulch, D. L., Drysdale, D. D., Horne, D. G., and Lloyd, A. C., Evaluated Kinetic Data for High Temperatures Reactions, Butterworths, London, 1972, vol. 1.

26. Dixon-Lewis, G., and Williams, D. J., in Comprehensive Chemical Kinetics (C. H. Bamford and C. F. H Tipper, Eds.), Elsevier, London, 1977, vol. 17, pp. 109-144.

27. Warnatz, J., in Combustion Chemistry (W. C. Gardiner, Ed.), Springer, New York, 1984, pp. 197-360.

28. Mansten, D. A., Hanson, R. K., and Bowman, C. T., J. Phys. Chem. 94:7119-7128 (1990).
29. Yetter, R. A., Dryer, F. L., and Rabitz, H., Combust. Sci. Technol. 79:97-128 (1991).

30. Clavin, P., Prog. Ener. Combust. Sci. 11:1-59 (1985).

31. Seshadri, K., Peters, N., and Williams, F. A., Combust. Flame 96:407-427 (1994).

32. Sánchez, A. L., Ph.D. Thesis, University of California, San Diego, La Jolla, CA, 1995.

33. Keyes, D. E., and Smooke, M., J. Computat. Phys. 73:267-288 (1987).

34. Smooke, M. D., Puri, I. K., and Seshadri, K., TwentySecond Symposium (International) on Combustion, The Combustion Institute, Pittsburgh, 1988, pp. 1555-1563.

35. Kee, R. J., Warnatz, J., and Miller, J. A., Technical Report UC-32, SAND83-8209, Sandia, Livermore, CA (1983)

36. Giovangigli, V., and Smooke, M. D., Appl. Numerical Math. 5:305-331 (1989).

37. Williams, F. A., Combustion Theory, Benjamin Cummings, Menlo Park, CA, 2nd edition, 1985.

38. Hirschfelder, J. O., Curtiss, C. F., and Bird, R. B., Molecular Theory of Gases and Liquids, Wiley, New York, 1954.

39. Kim, J. S., and Williams, F. A., Combust. Sci. Technol. 73:575-588 (1990).

40. Hoffman, J. D., Numerical Methods for Engineers and Scientists, McGraw-Hill, New York, 1992, pp. 358-361.

41. Abramowitz, M., and Stegun, A., Handbook of Mathematical Functions, Dover, New York, 1965.

Received 11 May 1995; revised 24 October 1995 